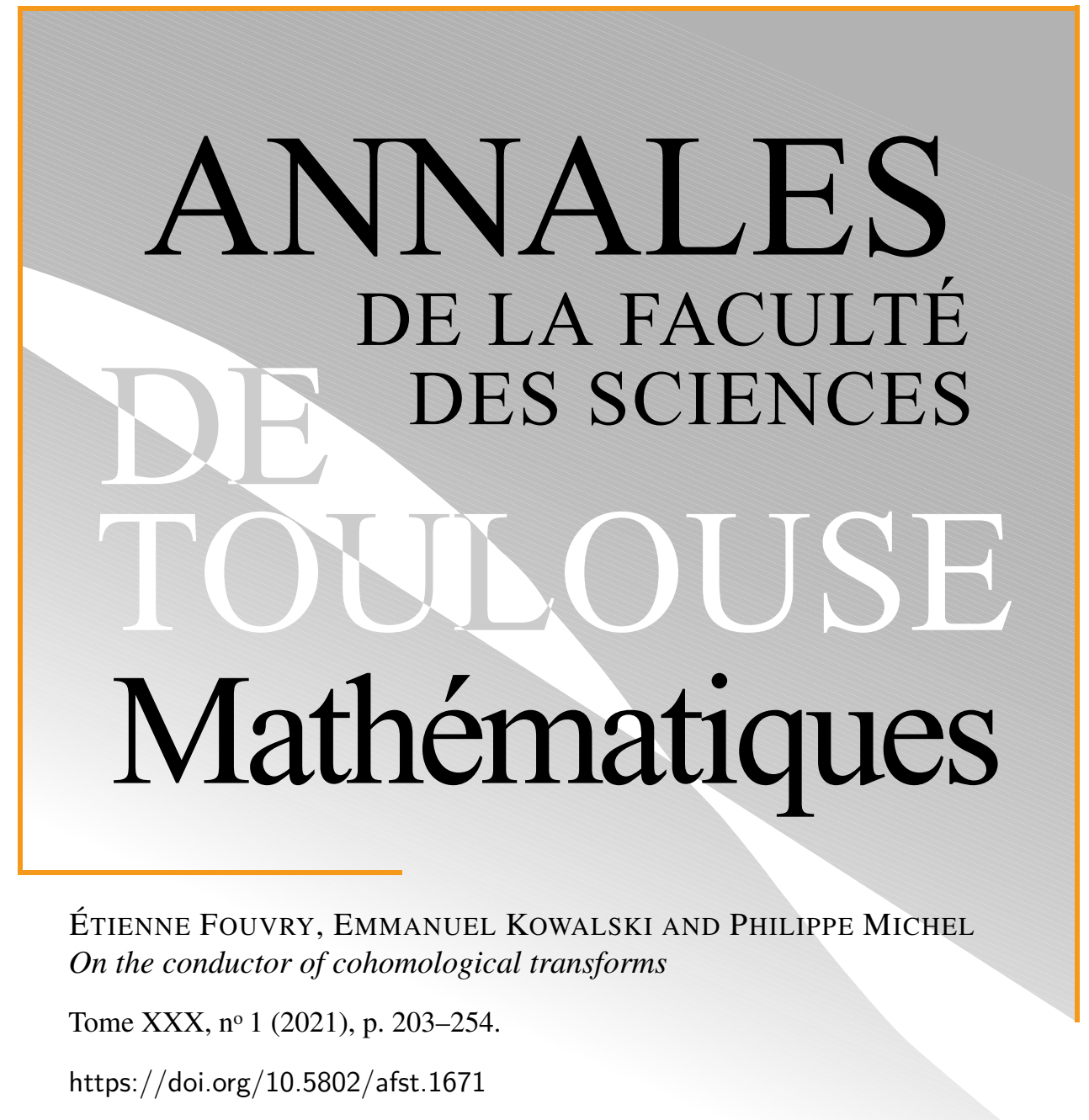

(c) Université Paul Sabatier, Toulouse, 2021.

L'accès aux articles de la revue «Annales de la faculté des sciences de Toulouse Mathématiques » (http://afst.centre-mersenne.org/) implique l'accord avec les conditions générales d'utilisation (http://afst.centre-mersenne.org/legal/). Les articles sont publiés sous la license CC-BY 4.0.

(c) $\underset{\mathrm{Br}}{\mathrm{Br}}$

Publication membre du centre

Mersenne pour l'édition scientifique ouverte MERSENNE http://www.centre-mersenne.org/ 


\title{
On the conductor of cohomological transforms ${ }^{(*)}$
}

\author{
Étienne Fouvry ${ }^{(1)}$, \\ Emmanuel Kowalski ${ }^{(2)}$ And Philippe Michel ${ }^{(3)}$
}

\begin{abstract}
In the analytic study of trace functions of $\ell$-adic sheaves over finite fields, a crucial issue is to control the conductor of sheaves constructed in various ways. We consider cohomological transforms on the affine line over a finite field which have trace functions given by linear operators with an additive character of a rational function in two variables as a kernel. We prove that the conductor of such transforms is bounded in terms of the complexity of the input sheaf and of the rational function defining the kernel, and discuss applications of this result, including motivating examples arising from the POLYMath8 project.

RÉsumé. - Dans l'étude analytique des fonctions traces de faisceaux $\ell$-adiques sur les corps finis, un problème crucial est de contrôler la taille du conducteur de faisceaux construits de façons variées. Nous considérons les transformées cohomologiques sur la droite affine sur un corps fini dont les fonctions traces sont données par des opérateurs linéaires dont la matrice est un caractère additif évalué sur une fonction rationnelle en deux variables. Nous prouvons que le conducteur de telles transformées est borné en fonction du conducteur du faisceau de départ et de la fraction rationnelle définissant le noyau. Enfin nous présentons des applications et des exemples, en particulier des exemples provenant du projet POLYMATH8.
\end{abstract}

$\left.{ }^{*}\right)$ Reçu le 21 janvier 2019, accepté le 16 juin 2019.

Keywords: Étale cohomology, conductor, $\ell$-adic sheaves, Riemann Hypothesis over finite fields, exponential sums.

2020 Mathematics Subject Classification: 11T23, 14F20, 11G20.

(1) Université Paris-Saclay, CNRS, Laboratoire de Mathématiques d'Orsay, 91405

Orsay, France - etienne.fouvry@universite-paris-saclay.fr

(2) ETH Zürich - D-MATH, Rämistrasse 101, CH-8092 Zürich, Switzerland kowalski@math.ethz.ch

(3) EPFL/SB/TAN, Station 8, CH-1015 Lausanne, Switzerland philippe.michel@epfl.ch

Ph. M. was partially supported by the SNF (grant 200021-137488) and the ERC (Advanced Research Grant 228304). É.F. thanks ETH Zürich, EPF Lausanne and the Institut Universitaire de France for financial support. Ph.M. and E.K. were supported by the SNF-DFG Grant 200020L_175755.

Article proposé par Damian Rössler. 


\section{Introduction}

This paper considers a problem which appeared in special cases in $[4,5,6]$ in our study of analytic applications of trace functions over finite fields. We are given a constructible $\ell$-adic sheaf $\mathcal{K}$ on $\mathbf{A}^{1} \times \mathbf{A}^{1}$ (or, potentially, on another algebraic surface) over a finite field $\mathbf{F}_{q}$, and we use it to define a "cohomological transform" with "kernel" $\mathcal{K}$, that maps a constructible $\ell$-adic sheaf $\mathcal{F}$ on $\mathbf{A}_{\mathbf{F}_{q}}^{1}$ to

$$
T_{\mathcal{K}}(\mathcal{F})=R^{1} p_{1, !}\left(p_{2}^{*} \mathcal{F} \otimes \mathcal{K}\right)(1 / 2),
$$

where $p_{1}, p_{2}$ are the two projections $p_{i}: \mathbf{A}^{1} \times \mathbf{A}^{1} \rightarrow \mathbf{A}^{1}$. The problem is then to estimate the conductor of $T_{\mathcal{K}}(\mathcal{F})$, as defined in [5], in terms of that of $\mathcal{F}$.

The arithmetic interpretation of this problem, and our motivation, is that for suitable input sheaves $\mathcal{F}$ (as described later in more detail), the trace function $t_{T_{\mathcal{K}}(\mathcal{F})}$ of is related to the trace functions $t_{\mathcal{K}}$ and $t_{\mathcal{F}}$ by

$$
t_{T_{\mathcal{K}}(\mathcal{F})}(x)=-\frac{1}{\sqrt{q}} \sum_{y \in \mathbf{F}_{q}} t_{\mathcal{F}}(y) t_{\mathcal{K}}(x, y),
$$

for all $x \in \mathbf{F}_{q}$. In other words, for all $\mathcal{F}$, we have $t_{T_{\mathcal{K}}(\mathcal{F})}=\mathrm{T}_{K}\left(t_{\mathcal{F}}\right)$, where

$$
K(x, y)=t_{\mathcal{K}}(x, y)
$$

and $\mathrm{T}_{K}$ is the (normalized) linear map defined on the space $C\left(\mathbf{F}_{q}\right)$ of complex-valued functions on $\mathbf{F}_{q}$ by the kernel $K$, i.e.,

$$
\mathrm{T}_{K}(\varphi)(x)=-\frac{1}{\sqrt{q}} \sum_{y \in \mathbf{F}_{q}} K(x, y) \varphi(y) .
$$

The most important example of such transforms arises for $K(x, y)=$ $\psi(x y)$, where $\psi$ is a non-trivial additive character, which corresponds to $\mathcal{K}=\mathcal{L}_{\psi(X Y)}\left(\right.$ where $X, Y$ are the coordinates on $\left.\mathbf{A}^{1} \times \mathbf{A}^{1}\right)$ : the corresponding linear operator $\mathrm{T}_{K}$ on trace functions is (minus the) normalized Fourier transform (which we denote also $\mathrm{FT}_{\psi}$ ) on $C\left(\mathbf{F}_{q}\right)$, namely

$$
\mathrm{FT}_{\psi}(\varphi)(x)=-\frac{1}{\sqrt{p}} \sum_{y \in \mathbf{F}_{p}} \varphi(y) \psi(x y) .
$$

The sheaf-theoretic construction, in that case, is due to Deligne, and it was extensively studied by Laumon [17].

This special case is crucial in [5] (and the following papers). In particular, it is essential for our applications that we have an estimate for the conductor 
of the Fourier transform $\mathcal{G}$ in terms only of the conductor of $\mathcal{F}$, which follows from the estimate

$$
\mathbf{c}(\mathcal{G}) \leqslant 10 \mathbf{c}(\mathcal{F})^{2}
$$

proved in [5, Prop. 8.2]. In order to establish this result, which we view as a form of "continuity" of the sheaf-theoretic Fourier transform, we used the deep theory of the local Fourier transform of Laumon [10, 17].

The general case of these transforms is a natural approach to estimates for two-variable character sums (and more complicated algebraic sums) based on Deligne's work, and an estimate for the conductor leads for instance easily to strong statements of "control of the diagonal" (see Proposition 5.7 for a precise statement).

It is not at all clear if a local theory like Laumon's applies to the general transforms we consider (the same applies to the theory of "affine cohomological transforms" of Katz [11], which has diophantine applications to stratification results for sums of trace functions). Thus, our present goal is to prove estimates for more general cohomological transforms. These will be weaker than (1.3), but more accessible. We will be able to do so when the kernel $\mathcal{K}$ is a rather general Artin-Schreier sheaf, or in other words (in the case when $q=p$ is prime) when

$$
K(x, y)=e\left(\frac{f(x, y)}{p}\right)
$$

for some rational function $f \in \mathbf{F}_{p}(X, Y)$.

The precise statement is given in Theorem 2.3 in the next section. In the case of the Fourier transform, this gives a form of the important property (1.3) which is more accessible than Laumon's theory. Section 14 treats this case fully, in order to motivate and clarify the algebraic tools used in the general case. In Section 5, we discuss some first applications of these bounds; for instance, we show how the ideas lead to an account of the character sums considered by Conrey and Iwaniec in [1]. This section can, to a large extent, be read independently of the part of the paper where the main results are proved.

\section{Remark 1.1.}

(1) W. Sawin has developed a much more general and powerful theory of complexity for (complexes of) $\ell$-adic sheaves on quasi-projective schemes, including all so-called 6 operations on the derived category. His work (see [22]) subsumes ours entirely, but involves much deeper algebraic geometry. The present paper may still be helpful for readers with a more analytic background as an illustration of fairly simple manipulations of the formalism of étale cohomology. 
(2) In recent work, I. Petrow and M. Young [19] generalized the estimate of Conrey and Iwaniec to more general characters. They need to estimate slightly different sums than those in [1], and the first draft of their preprint refered to this paper for this purpose. W. Sawin has also observed that their sums (as those of Conrey and Iwaniec) and special cases of hypergeometric sums, can be directly estimated by a simple appeal to Katz's book [10].

(3) A slightly different definition of the conductor suggested by W. Sawin leads to better estimates (e.g., a linear bound for the conductor of the Fourier transform instead of (1.3)). Since our work is in any case very restricted (see Remark (1)), and the most important qualitative feature is not affected for applications, we have not incorporated all the changes required by this adjustment.

\section{Acknowledgments}

Part of the original motivation for this paper in 2013/2104 arose in online discussions related to the POLYMath8 project.

We thank the referee for a detailed reading of the paper which led to significant simplications in several parts of the argument, and pointed out a mistake in one of our applications.

\section{Notation}

By "sheaf", or " $\ell$-adic sheaf", we will always mean "constructible $\overline{\mathbf{Q}}_{\ell^{-}}$ sheaf", where $\ell$ will be a prime number different from the characteristic of the base field.

For a power $q \neq 1$ of a prime $p$ and any integer $w \in \mathbf{Z}$, a $q$-Weil number of weight $w$ is an algebraic number $\alpha \in \mathbf{C}$ such that all Galois-conjugates $\beta$ of $\alpha$ satisfy $|\beta|=q^{w / 2}$.

An algebraic variety over a field $k$ is a finite type, separated, reduced scheme over $k$. If $X / k$ is an algebraic variety over a field $k$, and $\bar{k}$ is an algebraic closure of $k$, we denote by $\bar{X}$ or $X_{\bar{k}}$ the base change $X \times \bar{k}$.

For an algebraic variety $X$ over $\mathbf{F}_{q}$ and an $\ell$-adic sheaf $\mathcal{F}$ on $X$, we denote

$$
\chi_{c}(U, \mathcal{F})=\sum_{i=0}^{2 \operatorname{dim}(X)}(-1)^{i} \operatorname{dim} H_{c}^{i}\left(U \times \overline{\mathbf{F}}_{q}, \mathcal{F}\right) .
$$


If $X$ is the affine line, we will abbreviate $\chi(\mathcal{F})=\chi_{c}\left(\mathbf{A}^{1}, \mathcal{F}\right)$, and we write

$$
h^{1}(\mathcal{F})=\operatorname{dim} H_{c}^{1}\left(\mathbf{A}_{\overline{\mathbf{F}}_{q}}^{1}, \mathcal{F}\right) .
$$

If $X_{k}$ is an algebraic variety over a field $k$ and $x \in X(k)$, we denote by $\bar{x}$ a geometric point above $x$. If $k$ is algebraically closed, we take $\bar{x}=x$. If $\mathcal{F}$ is an étale sheaf on $X$, then $\mathcal{F}_{\bar{x}}$ denotes the stalk of $\mathcal{F}$ at $\bar{x}$.

Whenever a prime $\ell$ is given, we assume fixed an isomorphism $\iota: \overline{\mathbf{Q}}_{\ell} \rightarrow$ $\mathbf{C}$, and we use it as an implicit identification.

For any $\ell$-adic sheaf $\mathcal{F}$ on an algebraic variety $X_{\mathbf{F}_{q}}$, we write $t_{\mathcal{F}}(x)$ for the value at $x$ of the trace function of $\mathcal{F}$, i.e., we have

$$
t_{\mathcal{F}}(x)=\iota\left((\operatorname{tr} \mathcal{F})\left(\mathbf{F}_{q}, x\right)\right),
$$

the trace of the Frobenius of $\mathbf{F}_{q}$ acting on the stalk of $\mathcal{F}$ at $x$.

If $k / \mathbf{F}_{q}$ is a finite extension, we write

$$
t_{\mathcal{F}}(x,|k|)=t_{\mathcal{F}}(x, k)=\iota((\operatorname{tr} \mathcal{F})(k, x)) .
$$

\section{Statement of the main result}

We first recall the definition of the conductor of a constructible $\ell$-adic sheaf $\mathcal{F}$ on the affine line over a finite field $\mathbf{F}_{q}$. Indeed, since in this work it will be important to work with general constructible sheaves, and not only the middle-extension sheaves considered in our previous works, we need to adapt the definition slightly.

Let $\mathcal{F}$ be a constructible $\ell$-adic sheaf over $\mathbf{A}_{\mathbf{F}_{q}}^{1}$. Let $U \subset \mathbf{P}^{1}$ be the maximal dense open subset where $\mathcal{F}$ is lisse. Let $j: U \hookrightarrow \mathbf{P}^{1}$ be the corresponding open immersion. Recall that there is a canonical (adjunction) map

$$
\mathcal{F} \longrightarrow j_{*} j^{*} \mathcal{F}
$$

and that $\mathcal{F}$ is said to be a middle-extension sheaf if this is an isomorphism. In general, if we let

$$
\mathcal{F}_{0}=j_{*} j^{*} \mathcal{F}
$$

then one shows that $\mathcal{F}_{0}$ is a middle-extension sheaf on $\mathbf{P}_{\mathbf{F}_{q}}^{1}$, which is isomorphic to $\mathcal{F}$ when restricted to $U$. We define the conductor $\mathbf{c}(\mathcal{F})$ of the sheaf $\mathcal{F}$ by the formula

$$
\mathbf{c}(\mathcal{F})=\operatorname{rank}\left(\mathcal{F}_{0}\right)+\sum_{x} \operatorname{Swan}_{x}\left(\mathcal{F}_{0}\right)+n(\mathcal{F})+\operatorname{pct}(\mathcal{F}),
$$

where: 
- $n(\mathcal{F})=\left|\left(\mathbf{P}^{1}-U\right)\left(\overline{\mathbf{F}}_{q}\right)\right|$ is the number of singularities of $\mathcal{F}$ in $\mathbf{P}^{1}\left(\overline{\mathbf{F}}_{q}\right)$;

- the sum is over $\mathbf{P}^{1}\left(\overline{\mathbf{F}}_{q}\right)$, with all but finitely many terms vanishing;

- we define

$$
\operatorname{pct}(\mathcal{F})=\operatorname{dim} H_{c}^{0}\left(\mathbf{A}^{1} \times \overline{\mathbf{F}}_{q}, \mathcal{F}\right) .
$$

Remark 2.1.

(1) If $\mathcal{F}$ is a middle-extension sheaf on $\mathbf{A}_{\mathbf{F}_{q}}^{1}$, we have $\mathcal{F}=\mathcal{F}_{0}\left(\right.$ on $\left.\mathbf{A}^{1}\right)$ and

$$
\mathbf{c}(\mathcal{F})=\operatorname{rank}(\mathcal{F})+\sum_{x} \operatorname{Swan}_{x}(\mathcal{F})+n(\mathcal{F}),
$$

as in our previous works.

(2) Let $\mathcal{P}$ be the kernel of the map

$$
\mathcal{F} \longrightarrow j_{*} j^{*} \mathcal{F} \text {. }
$$

Then $\mathcal{P}$ has finite support; if this support is $S \subset \mathbf{A}^{1}\left(\overline{\mathbf{F}}_{q}\right)$, then

$$
|S| \leqslant \operatorname{pct}(\mathcal{F}) \leqslant \sum_{s \in S} \operatorname{dim} \mathcal{F}_{\bar{s}}
$$

(see $[8, \S 4.4,4.5]$ for a discussion).

(3) Note that $n(\mathcal{F})$ takes into account the fact that a general constructible sheaf might have "artificial" singularities, which are not singularities of the associated middle-extension sheaf. These may also be seen as the contribution to the conductor of the cokernel $\mathcal{F}$ of the map

$$
\mathcal{F} \longrightarrow j_{*} j^{*} \mathcal{F}
$$

which is also a sheaf with finite support.

For instance, let $U=\mathbf{P}^{1}-\mathbf{A}^{1}\left(\mathbf{F}_{p}\right)$ over $\mathbf{F}_{p}$, and let

$$
j: U \longrightarrow \mathbf{P}^{1}
$$

be the open immersion. Consider

$$
\mathcal{F}=j_{!} \overline{\mathbf{Q}}_{\ell},
$$

the extension by zero to $\mathbf{P}^{1}$ of the trivial sheaf on $U$. Then $\mathcal{F}_{0}$ is the trivial sheaf on $\mathbf{P}^{1}$, with $n\left(\mathcal{F}_{0}\right)=0$, and $\mathbf{c}\left(\mathcal{F}_{0}\right)=1$, while $\mathbf{c}(\mathcal{F})=1+n(\mathcal{F})=1+\left|\mathbf{A}^{1}\left(\mathbf{F}_{p}\right)\right|=p+1$ because of the artificial singularities created at the points in $\mathbf{A}^{1}\left(\mathbf{F}_{p}\right)$. It is necessary here to have a big conductor if we want some basic qualitative features of the Riemann Hypothesis to hold.

We note the following useful property:

$$
\mathbf{c}\left(\mathcal{F}_{1} \oplus \mathcal{F}_{2}\right) \leqslant \mathbf{c}\left(\mathcal{F}_{1}\right)+\mathbf{c}\left(\mathcal{F}_{2}\right)
$$


for two constructible sheaves on $\mathbf{A}^{1}$ (more generally, if

$$
0 \longrightarrow \mathcal{F}_{1} \longrightarrow \mathcal{F}_{3} \longrightarrow \mathcal{F}_{2} \longrightarrow 0
$$

is a short exact sequence of constructible sheaves on $\mathbf{A}^{1}$, then we have

$$
\mathbf{c}\left(\mathcal{F}_{3}\right) \leqslant \mathbf{c}\left(\mathcal{F}_{1}\right)+\mathbf{c}\left(\mathcal{F}_{2}\right)
$$

as one can check).

We also recall the definition of the drop of a constructible sheaf $\mathcal{F}$ on $\mathbf{A}_{\overline{\mathbf{F}}_{q}}^{1}$ at a point $x \in \mathbf{A}^{1}\left(\overline{\mathbf{F}}_{q}\right)$ : we have

$$
\operatorname{drop}_{x}(\mathcal{F})=\operatorname{rank}\left(\mathcal{F}_{0}\right)-\operatorname{dim} \mathcal{F}_{x},
$$

where $\mathcal{F}_{x}$ is the stalk of $\mathcal{F}$ at $x$. Note that the rank of $\mathcal{F}_{0}$ is also the "generic" rank of $\mathcal{F}$, i.e., the dimension of the fiber at a geometric generic point.

As mentioned in the introduction, we consider in this paper a kernel $\mathcal{K}$ which is an Artin-Schreier sheaf, with trace function given by additive characters of rational function. We give a formal definition to avoid any ambiguity concerning the behavior at the poles or points of indeterminacy of a rational function in two variables.

Definition 2.2 (Artin-Schreier sheaf on $\mathbf{A}^{n}$ ). - Let $\mathbf{F}_{q}$ be a finite field of characteristic $p$, and let $\ell \neq p$ be a prime number, and $\psi$ a non-trivial additive $\ell$-adic character of $\mathbf{F}_{q}$. Let $\mathcal{L}_{\psi}$ denote the associated Artin-Schreier sheaf on $\mathbf{A}_{\mathbf{F}_{q}}^{1}$ (see [2, Sommes Trig.] for the precise definition).

Let $f \in \mathbf{F}_{q}\left(X_{1}, \ldots, X_{n}\right)$ be a rational function for some $n \geqslant 1$. Write $f=f_{1} / f_{2}$ where $f_{i} \in \mathbf{F}_{q}\left[X_{1}, \ldots, X_{n}\right]$ and where $f_{1}$ is coprime with $f_{2}$. Let $U \subset \mathbf{A}^{n}$ be the open set where $f_{2}$ is invertible, $j: U \hookrightarrow \mathbf{A}^{n}$ the corresponding open immersion, and let

$$
f_{U}: U \longrightarrow \mathbf{A}^{1}
$$

be the morphism associated to the restriction of $f$ to $U$.

The Artin-Schreier sheaf on $\mathbf{A}^{n}$ associated to $f$ is the constructible $\ell$-adic sheaf on $\mathbf{A}_{\mathbf{F}_{q}}^{n}$ given by

$$
\mathcal{L}_{\psi(f)}=j_{!} f_{U}^{*} \mathcal{L}_{\psi}
$$

We also write $\mathcal{L}_{\psi\left(f\left(X_{1}, \ldots, X_{n}\right)\right)}$ for this sheaf. We define its conductor to be

$$
\mathbf{c}\left(\mathcal{L}_{\psi(f)}\right)=1+\operatorname{deg}\left(f_{1}\right)+\operatorname{deg}\left(f_{2}\right),
$$

and we will also sometimes just speak of the conductor $\mathbf{c}(f)$ of $f$.

We will find a satisfactory generalization of (1.3) for transforms associated to a kernel which is an Artin-Schreier sheaf. 
Theorem 2.3 (Conductor of Artin-Schreier transforms). - Let $\mathbf{F}_{q}$ be a finite field of order $q$ and characteristic $p, \ell$ a prime distinct from $p$. Let $\mathcal{K}$ be an $\ell$-adic sheaf on $\mathbf{A}^{1} \times \mathbf{A}^{1}$ over $\mathbf{F}_{q}$ of the form

$$
\mathcal{K}=\mathcal{L}_{\psi(f(X, Y))}
$$

where $\psi$ is a non-trivial additive $\ell$-adic character and $f \in \mathbf{F}_{q}(X, Y)$ is a rational function with conductor $<p$.

For constructible sheaves $\mathcal{F}$ on $\mathbf{A}_{\mathbf{F}_{q}}^{1}$, and $0 \leqslant i \leqslant 2$, let

$$
T_{\mathcal{K}}^{i}(\mathcal{F})=R^{i} p_{1, !}\left(p_{2}^{*} \mathcal{F} \otimes \mathcal{K}\right) .
$$

Then $T_{\mathcal{K}}^{i}(\mathcal{F})$ is constructible and there exists an integer $A \geqslant 1$ such that for any middle-extension sheaf $\mathcal{F}$ on $\mathbf{A}_{\mathbf{F}_{q}}^{1}$, and $0 \leqslant i \leqslant 2$, we have

$$
\mathbf{c}\left(T_{\mathcal{K}}^{i}(\mathcal{F})\right) \leqslant(2 \mathbf{c}(\mathcal{K}) \mathbf{c}(\mathcal{F}))^{A} .
$$

In particular, if $f$ is obtained by reduction modulo $p$ of a fixed nonconstant rational function $f_{1} / f_{2}$, where $f_{i} \in \mathbf{Z}[X, Y]$, and if we have some integer $M \geqslant 1$ and, for each $p$, we consider a sheaf $\mathcal{F}_{p}$ modulo $p$ with conductor $\leqslant M$, then we have

$$
\mathbf{c}\left(T_{\mathcal{K}}^{1} \mathcal{F}_{p}\right) \ll 1
$$

for all primes. This allows us to apply all our estimates for trace functions to the trace functions of these sheaves; we give some examples in Section 5.

Remark 2.4.

(1) The fact that the sheaf $T_{\mathcal{K}}^{i}(\mathcal{F})$ is constructible for any constructible sheaf $\mathcal{F}$ and all $i$ follows from [2, Arcata, IV, Thm. 6.2] (see also [7, Thm. 7.8.1]).

(2) Note that we omitted the Tate twist in this statement, since it concerns purely geometric facts.

We need to consider all the transforms $T_{\mathcal{K}}^{i}$, and not only $T_{\mathcal{K}}^{1}$ because this will turn out to be useful in the proof, which is interleaved with the proof of the following other useful fact:

TheOREm 2.5 (Bounds for Betti numbers). - Let $\mathbf{F}_{q}$ be a finite field of order $q$ and characteristic $p, \ell$ a prime distinct from $p$. Let $\mathcal{K}$ be an $\ell$-adic sheaf on $\mathbf{A}^{1} \times \mathbf{A}^{1}$ over $\mathbf{F}_{q}$ of the form

$$
\mathcal{K}=\mathcal{L}_{\psi(f(X, Y))}
$$

where $\psi$ is a non-trivial additive $\ell$-adic character and $f \in \mathbf{F}_{q}(X, Y)$ is a rational function with conductor $<p$. 
There exists an integer $B \geqslant 1$ such that for any middle-extension sheaf $\mathcal{F}$ on $\mathbf{A}_{\mathbf{F}_{q}}^{1}$ and for $0 \leqslant i \leqslant 4$, we have

$$
\operatorname{dim} H_{c}^{i}\left(\mathbf{A}^{2} \times \overline{\mathbf{F}}_{q}, p_{2}^{*} \mathcal{F} \otimes \mathcal{K}\right) \leqslant(2 \mathbf{c}(f) \mathbf{c}(\mathcal{F}))^{B} .
$$

Roughly speaking, we will proceed as follows:

(1) we prove Theorem 2.3 for $\mathcal{F}$ the trivial sheaf, and observe that Theorem 2.5 is a known fact in that case, from bounds on Betti numbers due to Bombieri, Adolphson-Sperber and Katz [14];

(2) using Theorem 2.3 for the trivial sheaf, we first prove Theorem 2.5 for all input sheaves $\mathcal{F}$ and $i=2$;

(3) finally, we prove Theorem 2.3 in general and deduce Theorem 2.5 for all $i$.

\section{Diophantine motivation of the proof}

The arguments of the proof of Theorem 2.3 are purely algebraic and geometric, and exercise much of the basic formalism of étale cohomology, as well as a simple use of spectral sequences. However, there is a concrete analytic motivation from (expected) properties of sums of trace functions, and we will first present it. This is based on the Riemann Hypothesis over finite fields, and is similar in principle to the discussion [13, Lecture IV, Interlude] by Katz that motivates the crucial step in his paper.

The first ingredient is a lemma that, essentially, allows one to estimate, in terms of accessible global invariants, the conductor of a middle-extension sheaf, satisfying some conditions, assuming one already knows estimates for the rank and the number of singularities. In other words, it provides a bound for the sum of Swan conductors in global terms, assuming that the rank and number of singularities are under control.

To be slightly more precise, assume that $\mathcal{F}$ is a middle-extension sheaf on $\mathbf{A}_{\mathbf{F}_{q}}^{1}$ which is pointwise pure of weight 0 , and assume in addition the following conditions:

(1) $\mathcal{F}$ has no geometrically trivial Jordan-Hölder factor;

(2) the Frobenius action on $H_{c}^{1}(\bar{U}, \mathcal{F})$ is pure of weight 1 , for the maximal dense open set $U$ on which $\mathcal{F}$ is lisse.

We then define the invariant

$$
\widetilde{\sigma}(\mathcal{F})=\limsup _{\nu \rightarrow+\infty} \frac{\left|S_{\nu}(\mathcal{F})\right|}{q^{\nu / 2}},
$$


where

$$
S_{\nu}(\mathcal{F})=\sum_{x \in U\left(\mathbf{F}_{q^{\nu}}\right)} t_{\mathcal{F}}\left(x, q^{\nu}\right),
$$

for $\nu \geqslant 1$ (in other words, these are the sums of trace functions over extension fields). Then we have

$$
\mathbf{c}(\mathcal{F}) \leqslant 3 \operatorname{rank}(\mathcal{F})+n(\mathcal{F})+\widetilde{\sigma}(\mathcal{F}) .
$$

Indeed, using (1) and the notation Fr for the frobenius, the Lefschetz trace formula applied to $U$ over $\mathbf{F}_{q^{\nu}}$ gives

$$
S_{\nu}(\mathcal{F})=-\operatorname{tr}\left(\operatorname{Fr}^{\nu} \mid H_{c}^{1}(\bar{U}, \mathcal{F})\right),
$$

so that the purity assumption implies

$$
\tilde{\sigma}(\mathcal{F})=\operatorname{dim} H_{c}^{1}(\bar{U}, \mathcal{F})
$$

and then the stated bound follows from Lemma 4.11 below (which is an elementary application of the Euler-Poincaré formula).

We now consider the situation of Theorem 2.3. We will assume (and this is where the argument is not easy to make rigorous in a decent generality) that the sheaves $\mathcal{G}=T_{\mathcal{K}}(\mathcal{F})$ whose conductor we wish to control always satisfy the conditions above (i.e., that they are middle-extensions, pointwise of weight 0 , and (1), (2) hold). We first assume that we can find suitable estimates of the rank, of the number of singularities, and of the punctual part of $\mathcal{G}$ (intuitively, this is possible because these amounts to fiber-by-fiber considerations, which boil down to properties of one-variable sheaves, which are relatively wellunderstood; the case of the trivial sheaf $\mathcal{F}$ is quite elementary, but the details will turn out to be a bit involved in the general case). We then need to estimate $\tilde{\sigma}(\mathcal{G})$. For this purpose, we proceed in two steps.

In Step 1, we consider only the trivial input sheaf $\mathcal{F}=\overline{\mathbf{Q}}_{\ell}$. We then have

$$
\begin{aligned}
\frac{S_{\nu}(\mathcal{G})}{q^{\nu / 2}} & =-\frac{1}{q^{\nu}} \sum_{x \in \mathbf{F}_{q^{\nu}}}\left(\sum_{y \in \mathbf{F}_{q^{\nu}}} \psi_{\nu}(f(x, y))\right) \\
& =-\frac{1}{q^{\nu}} \sum_{(x, y) \in \mathbf{F}_{q^{\nu}} \times \mathbf{F}_{q^{\nu}}} \psi_{\nu}(f(x, y))
\end{aligned}
$$

(where $\psi_{\nu}=\psi \circ \operatorname{Tr}_{\mathbf{F}_{q \nu} / \mathbf{F}_{q}}$ ) and the two-variable character sum (under Assumption (2) for $\mathcal{G}$ ) has square-root cancellation, so that the bounds on Betti numbers of [14] (or often their predecessors, due to Bombieri and AdolphsonSperber) give

$$
\limsup _{\nu \rightarrow+\infty} \frac{\left|S_{\nu}(\mathcal{G})\right|}{q^{\nu / 2}} \leqslant C
$$

where $C \geqslant 1$ depends only on the conductor of $f$. 
In Step 2, we handle the case of a general sheaf $\mathcal{F}$. We then have

$$
\begin{aligned}
\frac{S_{\nu}(\mathcal{G})}{q^{\nu / 2}} & =-\frac{1}{q^{\nu}} \sum_{x \in \mathbf{F}_{q^{\nu}}} \sum_{y \in \mathbf{F}_{q^{\nu}}} t_{\mathcal{F}}\left(y, q^{\nu}\right) \psi_{\nu}(f(x, y)) \\
& =-\frac{1}{q^{\nu}} \sum_{y \in \mathbf{F}_{q^{\nu}}} t_{\mathcal{F}}\left(y, q^{\nu}\right) \sum_{x \in \mathbf{F}_{q^{\nu}}} \psi_{\nu}(f(x, y)) .
\end{aligned}
$$

The basic point is that this is the inner-product of the trace functions of the dual sheaf of $\mathcal{F}$ and of the sheaf $R^{1} p_{2, !} \mathcal{L}_{\psi(f(X, Y))}$. This last sheaf, by the first step (applied to $\mathcal{L}_{\psi(f(Y, X))}$ ), has conductor bounded by a constant depending only on the conductor of $f$. By assumption again, we have squareroot cancellation in this sum as $\nu \rightarrow+\infty$, and by the quasi-orthogonality formulation of Deligne's proof of the Riemann Hypothesis over finite fields [3], we obtain

$$
\widetilde{\sigma}(\mathcal{G})=\limsup _{\nu \rightarrow+\infty} \frac{\left|S_{\nu}(\mathcal{G})\right|}{q^{\nu / 2}} \leqslant C^{\prime},
$$

where $C^{\prime}$ depends only on the conductors of $\mathcal{F}$ and of $f$.

Remark 3.1. - In terms of linear operators and of the standard (unnormalized) inner-product on functions on $\mathbf{F}_{q}$, we exploit the obvious identity

$$
\sum_{x \in \mathbf{F}_{q}}\left(\mathrm{~T}_{K} \varphi\right)(x)=\left\langle\mathrm{T}_{K} \varphi, 1\right\rangle=\left\langle\varphi, \mathrm{T}_{K}^{*} 1\right\rangle,
$$

where the adjoint operator $\mathrm{T}_{K}^{*}$ has kernel $K^{*}(x, y)=\overline{K(y, x)}$; the first step in our sketch amounts to bounding (the complexity of) $\mathrm{T}_{K}^{*} 1$, and the second applies standard inequalities to deduce a bound for the sum over $x$.

In contrast with this sketch, the proof of Theorem 2.3 below is entirely algebraic and does not require the Riemann Hypothesis over finite fields. It also applies in greater generality, so that the assumptions (1) and (2) are not needed. Roughly speaking, instead of sums of trace functions, we control directly the dimension $\widetilde{\sigma}(\mathcal{G})$ of $H_{c}^{1}(\bar{U}, \mathcal{G})$ for the transformed sheaf $\mathcal{G}$. The "combination of sums" in (3.1) and the "exchange of order of summation" in (3.2) are replaced by arguments based on spectral sequences (compare again with [13, Lecture IV, Interlude], and the dictionary [2, Sommes Trig., $\S 2])$. The proof is however complicated by the fact that we must also control the possible punctual part of the transformed sheaf.

Before giving the proof, we will present some algebraic preliminaries and then discuss first the motivating applications in Section 5 (Section 4 may be skipped in a first reading, since Section 5 will only refer to it incidentally). We then set up the proof in Section 6, and follow by presenting an (almost) self-contained account of the Fourier transform and of the special case which 
is relevant to the PoLYmath8 project as it appears in [20] (see Section 14). Finally, we give the full proof of Theorems 2.3 and 2.5.

\section{Preliminaries}

We begin by some preliminary inequalities between the dimensions of the cohomology groups and the conductor of sheaves on the affine line.

\subsection{General results from étale cohomology}

We first state formally some properties of étale cohomology that we will often use.

Proposition 4.1.

(1) Let $f: Y_{k} \rightarrow X_{k}$ be a morphism of algebraic varieties over an algebraically closed field $k$, with fibers of dimension $\leqslant n$. Let $\mathcal{F}$ be a constructible $\ell$-adic sheaf on $Y$. We have $R^{i} f_{!} \mathcal{F}=0$ for $i<0$ and for $i>2 n$. In particular, if $\mathcal{F}$ is a sheaf on $X$ and $X$ has dimension $\leqslant n$, we have $H_{c}^{i}(X, \mathcal{F})=0$ for $i<0$ and for $i>2 n$.

(2) Let $X_{k}$ be an algebraic variety over an algebraically closed field $k$, let $U \subset X$ be an open subset and $C=X-U$ its complement. Let $\mathcal{F}$ be a constructible $\ell$-adic sheaf on $X$. We have a long sequence

$$
\cdots \longrightarrow H_{c}^{i}(U, \mathcal{F}) \longrightarrow H_{c}^{i}(X, \mathcal{F}) \longrightarrow H_{c}^{i}(C, \mathcal{F}) \longrightarrow H_{c}^{i+1}(U, \mathcal{F}) \longrightarrow \cdots
$$

and in particular, for all $i \geqslant 0$, we have

$$
\begin{aligned}
& \operatorname{dim} H_{c}^{i}(X, \mathcal{F}) \leqslant \operatorname{dim} H_{c}^{i}(U, \mathcal{F})+\operatorname{dim} H_{c}^{i}(C, \mathcal{F}) \\
& \operatorname{dim} H_{c}^{i}(U, \mathcal{F}) \leqslant \operatorname{dim} H_{c}^{i}(X, \mathcal{F})+\operatorname{dim} H_{c}^{i-1}(C, \mathcal{F}) .
\end{aligned}
$$

(3) Let $X_{k}$ be a smooth affine algebraic variety over an algebraically closed field $k$, pure of dimension $n \geqslant 0$, and let $\mathcal{F}$ be a lisse $\ell$-adic sheaf on $X$. We have

$$
H_{c}^{i}(X, \mathcal{F})=0
$$

for $0 \leqslant i<n$.

(4) Let $f: X_{k} \rightarrow Y_{k}$ be a morphism of algebraic varieties over an algebraically closed field $k$, and let $\mathcal{F}$ be an $\ell$-adic constructible sheaf on $X$. Then, for $y \in Y$ and $i \geqslant 0$, the stalk of $R^{i} f_{!} \mathcal{F}$ at $y$ is naturally isomorphic to $H_{c}^{i}\left(f^{-1} X, \mathcal{F}\right)$. 
Proof. - (1) is the cohomological dimension property; the vanishing of $R^{i} f_{!} \mathcal{F}$ for $i<0$ is immediate by definition, while the vanishing for $i>2 n$ can be found, e.g., in [2, Arcata, IV, Thm. 6.1] or [7, Thm. 7.4.5]; the case of $H_{c}^{i}$ follows by considering $f: X \rightarrow \operatorname{Spec}(k)$, the structure morphism.

(2) is the so-called "excision" long-exact sequence, see for instance [2, Sommes Trig., (2.5.1)*]); the inequality (4.2) for a given $i \geqslant 0$ is an immediate consequence of the fragment

$$
H_{c}^{i}(U, \mathcal{F}) \longrightarrow H_{c}^{i}(X, \mathcal{F}) \longrightarrow H_{c}^{i}(C, \mathcal{F}),
$$

and (4.3) is a consequence of

$$
H_{c}^{i-1}(C, \mathcal{F}) \longrightarrow H_{c}^{i}(U, \mathcal{F}) \longrightarrow H_{c}^{i}(X, \mathcal{F}) .
$$

(3) is the property of affine cohomological dimension for lisse sheaves; it follows for instance from the Poincaré duality

$$
H_{c}^{i}(X, \mathcal{F}) \simeq H^{n-i}\left(X, \mathcal{F}^{*}\right)
$$

where $\mathcal{F}^{*}$ is the dual of $\mathcal{F}$ (see for instance [2, Sommes Trig., Remarque $1.18(\mathrm{c})]$; note that the right-hand side is a cohomology group with no restriction of compact support) and the vanishing property

$$
H^{i}(X, \mathcal{F})=0
$$

for an affine scheme $X$ and $i<\operatorname{dim}(X)$ (see, e.g., [2, Arcata, IV, Thm. 6.4]).

(4) is a special case of the proper base change theorem, (see, e.g., [2, Arcata, IV, Thm. 5.4] or [7, Thm. 7.4.4(i)]).

The following lemma will also be used frequently:

LEMmA 4.2. - Let $\mathcal{F}$ and $\mathcal{G}$ be middle-extension $\ell$-adic sheaves on $\mathbf{A}_{\mathbf{F}_{q}}^{1}$. Then we have

$$
H_{c}^{0}\left(\mathbf{A}^{1} \times \overline{\mathbf{F}}_{q}, \mathcal{F} \otimes \mathcal{G}\right)=0,
$$

i.e., the tensor product has no punctual part.

Proof. - In general, for a constructible sheaf $\mathcal{H}$ lisse on a dense open set $U \subset \mathbf{A}^{1}$, the condition

$$
H_{c}^{0}\left(\mathbf{A}^{1} \times \overline{\mathbf{F}}_{q}, \mathcal{H}\right)=0
$$

amounts to saying that, for all $x \in\left(\mathbf{A}^{1}-U\right)\left(\overline{\mathbf{F}}_{q}\right)$, the specialization map

$$
\mathcal{H}_{x} \longrightarrow \mathcal{H}_{\bar{\eta}}^{I_{x}}
$$

is injective (see $[8, \S 4.4]$ for instance), where $I_{\bar{x}}$ is the inertia group at $x$. We now have

$$
(\mathcal{F} \otimes \mathcal{G})_{x}=\mathcal{F}_{x} \otimes \mathcal{G}_{x} \hookrightarrow \mathcal{F}_{\bar{\eta}}^{I_{x}} \otimes \mathcal{G}_{\bar{\eta}}^{I_{x}} \subset\left(\mathcal{F}_{\bar{\eta}} \otimes \mathcal{G}_{\bar{\eta}}\right)^{I_{x}}=(\mathcal{F} \otimes \mathcal{G})_{\bar{\eta}}^{I_{x}} .
$$




\subsection{Basic bounds on the dimension of cohomology groups}

Another frequently-used fact, which is implicit in our previous work in the case of middle-extension sheaves, is the control of Betti numbers of constructible sheaves on $\mathbf{A}^{1}$ in terms of the conductor:

Lemma 4.3. - Let $\mathbf{F}_{q}$ be a finite field of characteristic $p$, let $\ell \neq p$ be a prime number and $\mathcal{F}$ an $\ell$-adic constructible sheaf on $\mathbf{A}_{\mathbf{F}_{q}}^{1}$. For $i=0,2$, we have

$$
\begin{aligned}
\operatorname{dim} H_{c}^{i}\left(\mathbf{A}^{1} \times \overline{\mathbf{F}}_{q}, \mathcal{F}\right) & \leqslant \mathbf{c}(\mathcal{F}) \\
\text { and } \quad \operatorname{dim} H_{c}^{1}\left(\mathbf{A}^{1} \times \overline{\mathbf{F}}_{q}, \mathcal{F}\right) & \leqslant 2 \mathbf{c}(\mathcal{F})+\mathbf{c}(\mathcal{F})^{2} .
\end{aligned}
$$

Proof. - For $i=0$, this is obvious from the definition of $\operatorname{pct}(\mathcal{F}) \leqslant \mathbf{c}(\mathcal{F})$. For $i=2$, we use the fact that if $\mathcal{F}$ is lisse on a dense open subset $U \subset \mathbf{A}^{1}$, we have

$$
H_{c}^{2}\left(\mathbf{A}^{1} \times \overline{\mathbf{F}}_{q}, \mathcal{F}\right)=H_{c}^{2}(\bar{U}, \mathcal{F}) \simeq\left(\mathcal{F}_{\bar{\eta}}\right)_{\pi_{1}(\bar{U}, \bar{\eta})},
$$

the coinvariant space for the action of the geometric fundamental group on the geometric generic fiber (see, e.g., [2, Sommes Trig., Rem. 1.18(d)]; the first equality is also a consequence of excision) and hence

$$
\operatorname{dim} H_{c}^{2}\left(\mathbf{A}^{1} \times \overline{\mathbf{F}}_{q}, \mathcal{F}\right) \leqslant \operatorname{rank}(\mathcal{F}) \leqslant \mathbf{c}(\mathcal{F}) .
$$

For $i=1$, we use the Euler-Poincaré formula (see $[9,8.5 .2,8.5 .3]$ ) to get

$$
\begin{aligned}
\operatorname{dim} H_{c}^{1}\left(\mathbf{A}^{1} \times \overline{\mathbf{F}}_{q}, \mathcal{F}\right) & \\
=-\operatorname{rank}(\mathcal{F})+ & \operatorname{dim} H_{c}^{0}\left(\mathbf{A}^{1} \times \overline{\mathbf{F}}_{q}, \mathcal{F}\right)+\operatorname{dim} H_{c}^{2}\left(\mathbf{A}^{1} \times \overline{\mathbf{F}}_{q}, \mathcal{F}\right) \\
& +\sum_{x}\left(\operatorname{drop}_{x}(\mathcal{F})+\operatorname{Swan}_{x}(\mathcal{F})\right)+\operatorname{Swan}_{\infty}(\mathcal{F})
\end{aligned}
$$

where the sum is over $x \in \mathbf{A}^{1}\left(\overline{\mathbf{F}}_{q}\right)$, and all but finitely many terms are zero, and the result follows from the definition of the conductor since $\operatorname{drop}_{x}(\mathcal{F})=$ $\operatorname{rank}(\mathcal{F})-\operatorname{dim} \mathcal{F}_{x} \leqslant \operatorname{rank}(\mathcal{F})$.

The following was also proved for middle-extensions in our previous works.

Lemma 4.4. - Let $\mathbf{F}_{q}$ be a finite field of characteristic $p$, let $\ell \neq p$ be a prime number and $\mathcal{F}_{1}$ and $\mathcal{F}_{2}$ be $\ell$-adic constructible sheaves on $\mathbf{A}_{\mathbf{F}_{q}}^{1}$. We have

$$
\mathbf{c}\left(\mathcal{F}_{1} \otimes \mathcal{F}_{2}\right) \leqslant 8 \mathbf{c}\left(\mathcal{F}_{1}\right)^{2} \mathbf{c}\left(\mathcal{F}_{2}\right)^{2}
$$

Proof. - One checks easily (as in [5, Prop. 8.2(2)]) that for the middleextension part $\left(\mathcal{F}_{1} \otimes \mathcal{F}_{2}\right)_{0}$ we have

$$
\mathbf{c}\left(\left(\mathcal{F}_{1} \otimes \mathcal{F}_{2}\right)_{0}\right) \leqslant 6 \mathbf{c}\left(\mathcal{F}_{1}\right)^{2} \mathbf{c}\left(\mathcal{F}_{2}\right)^{2}
$$


and as for the punctual part, we have

$$
\operatorname{pct}\left(\mathcal{F}_{1} \otimes \mathcal{F}_{2}\right) \leqslant\left(n_{1}+n_{2}\right) m_{1} m_{2}
$$

where $n_{i}$ is the number of points where there are punctual sections of $\mathcal{F}_{i}$, while $m_{i}$ is the maximal dimension of the space of sections supported at a single point. Since

$$
\begin{aligned}
\left(n_{1}+n_{2}\right) m_{1} m_{2} & \leqslant\left(\mathbf{c}\left(\mathcal{F}_{1}\right)+\mathbf{c}\left(\mathcal{F}_{2}\right)\right) m_{1} m_{2} \\
& \leqslant 2 \mathbf{c}\left(\mathcal{F}_{1}\right) \mathbf{c}\left(\mathcal{F}_{2}\right) m_{1} m_{2} \\
& \leqslant 2 \mathbf{c}\left(\mathcal{F}_{1}\right)^{2} \mathbf{c}\left(\mathcal{F}_{2}\right)^{2}
\end{aligned}
$$

we get the result.

\subsection{Number of singularities}

We will also use a criterion to bound the number of singularities in terms of estimates for the punctual part.

LEMMA 4.5. - Let $\mathbf{F}_{q}$ be a finite field of characteristic $p, \ell \neq p$ a prime number and $\mathcal{F}$ an $\ell$-adic constructible sheaf on $\mathbf{A}_{\mathbf{F}_{q}}^{1}$. Let $U \subset \mathbf{A}^{1}$ be a dense open set such that the dimension of the stalks $\mathcal{F}_{x}$ is constant, equal to some integer $d \geqslant 0$, for all $x \in U\left(\overline{\mathbf{F}}_{q}\right)$. We then have

$$
n(\mathcal{F}) \leqslant\left|\left(\mathbf{P}^{1}-U\right)\left(\overline{\mathbf{F}}_{q}\right)\right|+\operatorname{pct}(\mathcal{F}) .
$$

Proof. - Since $U$ contains the generic point $\eta$ of $\mathbf{A}^{1}$, we have

$$
\operatorname{rank}(\mathcal{F})=\operatorname{dim} \mathcal{F}_{\bar{\eta}}=d
$$

Let $U_{1} \subset U$ be the open dense subset where $\mathcal{F}$ is lisse, and let $x \in$ $\left(U-U_{1}\right)\left(\overline{\mathbf{F}}_{q}\right)$, i.e., a point of $U$ where $\mathcal{F}$ is not lisse. Let $\varphi: \mathcal{F}_{x} \rightarrow \mathcal{F}_{\bar{\eta}}^{I_{x}}$ be the canonical map. The image has dimension $<d$ (since otherwise, for dimension reasons, $I_{x}$ would act trivially on the geometric generic fiber $\mathcal{F}_{\bar{\eta}}$, and $\mathcal{F}$ would be lisse at $x$ ), and since $\operatorname{dim} \mathcal{F}_{x}=d$, it follows that

$$
\operatorname{dim} \operatorname{ker} \varphi \geqslant 1
$$

which means that $x$ is in the support of the punctual part of $\mathcal{F}$. Thus the number of such $x$ is at most the size of this support, which is bounded by $\operatorname{pct}(\mathcal{F})$. Adding the points of $\left(\mathbf{P}^{1}-U\right)\left(\overline{\mathbf{F}}_{q}\right)$ leads to the result. 


\subsection{Application to Artin-Schreier sheaves}

Given $g \in \mathbf{F}_{q}(X)$ a non-constant rational function in one variable let $\mathcal{L}=\mathcal{L}_{\psi(g(X))}$ its associated Artin-Schreier sheaf. The next lemma recalls the crucial link between the Swan conductor of $\mathcal{L}$ at a given point and its order as a pole of $g$.

LEMMA 4.6. - Let $\mathbf{F}_{q}$ be a finite field of order $q$ and characteristic $p, \ell \neq$ $p$ a prime number. Let $g \in \mathbf{F}_{q}(X)$ be a non-constant rational function and $\mathcal{L}$ its associated Artin-Schreier sheaf. For $x \in \mathbf{P}^{1}\left(\overline{\mathbf{F}}_{q}\right)$, the Swan conductor of $\mathcal{L}$ at $x$ is at most equal to the order of the pole of $g$ at $x$, and there is equality if the numerator and denominator of $g$ have degree $<p$.

Proof. - This is a standard property (see, for instance, [2, Sommes Trig., $(3.5 .4)])$.

We next discuss relations between two-variable Artin-Schreier sheaves and specializations of one variable. We need first some notation.

DEFinition 4.7 (Specializations). - Let $\mathbf{F}_{q}$ be a finite field of order $q$ and characteristic $p$, $\ell$ a prime distinct from $p$. Let $f \in \mathbf{F}_{q}(X, Y)$ be a non-constant rational function and let

$$
\mathcal{K}:=\mathcal{L}_{\psi(f(X, Y))}
$$

be the Artin-Schreier sheaf on $\mathbf{A}_{\mathbf{F}_{q}}^{2}$ associated to $f$. We introduce the further notations:

(1) If $x \in \overline{\mathbf{F}}_{q}$ is such that $X-x$ does not divide the denominator of $f$, we denote by $f_{x} \in \mathbf{F}_{q}(Y)$ the specialization $f(x, Y)$ of $f$.

(2) For every finite extension $k / \mathbf{F}_{q}$ and every $x \in k$, the specialization of $\mathcal{K}$ at $x$ is the $\ell$-adic constructible sheaf on $\mathbf{A}_{k}^{1}$ given by

$$
\mathcal{K}_{x}=j_{x}^{*} \mathcal{K},
$$

where $j_{x}:\{x\} \times \mathbf{A}^{1} \hookrightarrow \mathbf{A}^{2}$ is the closed immersion.

These two definitions are related as follows:

LEMMA 4.8. - Let $\mathbf{F}_{q}$ be a finite field of order $q$ and characteristic $p$, $\ell \neq p$ a prime number. Let $\mathcal{K}=\mathcal{L}_{\psi(f(X, Y))}$ be an $\ell$-adic Artin-Schreier sheaf on $\mathbf{A}^{2}$ over $\mathbf{F}_{q}$, where $f \in \mathbf{F}_{q}(X, Y)$ is a non-constant rational function.

(1) For any finite extension $k / \mathbf{F}_{q}$ and $x \in k$, we have

$$
\mathcal{K}_{x}=0
$$

if $X-x$ divides the denominator of $f$, and otherwise

$$
\begin{gathered}
\mathcal{K}_{x}=j_{!} \mathcal{L}_{\psi\left(f_{x}\right)} \\
-218-
\end{gathered}
$$


where $j: U_{x} \rightarrow\{x\} \times \mathbf{A}^{1}$ is the open immersion of the open subset of $\{x\} \times \mathbf{A}^{1}$ which is the intersection of $\{x\} \times \mathbf{A}^{1}$ and the open set of $\mathbf{A}^{2}$ where the denominator of $f$ is invertible.

If $\{x\} \times \mathbf{A}^{1}$ does not intersect the zero set of the numerator of $f$, then $\mathcal{K}_{x}$ is isomorphic to the Artin-Schreier sheaf $\mathcal{L}_{\psi\left(f_{x}\right)}$ associated to $f_{x}$.

(2) For every finite extension $k / \mathbf{F}_{q}$ and all $x \in k$, we have

$$
\mathbf{c}\left(\mathcal{K}_{x}\right) \leqslant 2 \mathbf{c}(f) \text {. }
$$

Proof.

(1). - If $X-x$ divides the denominator of $f$, then by definition the sheaf $\mathcal{K}$ is zero on $\{x\} \times \mathbf{A}^{1}$, and hence $\mathcal{K}_{x}=0$.

If $X-x$ does not divide the denominator of $f$, then there are only finitely many points where $\{x\} \times \mathbf{A}^{1}$ intersects the open set $U$ where the denominator is invertible. The sheaf $\mathcal{K}_{x}$ has zero stalk at these points, and is isomorphic to the one-variable sheaf $\mathcal{L}_{\psi(f(x, Y))}$ on the complementary open set, which is the result we claim.

If $\{x\} \times \mathbf{A}^{1}$ does not intersect the zero set of the numerator of $f$, then the points in $\{x\} \times \mathbf{A}^{1}$ where $\mathcal{K}_{x}$ has zero stalk are precisely the poles of $f_{x}$, which means that $j_{!} \mathcal{L}_{\psi\left(f_{x}\right)}=\mathcal{L}_{\psi\left(f_{x}\right)}$ as Artin-Schreier sheaf on $\mathbf{A}_{\mathbf{F}_{q}}^{1}$.

(2). - If $\mathcal{K}_{x}=0$, then the conductor bound is trivial, and otherwise we obtain from (1) the bound

$$
\begin{aligned}
\mathbf{c}\left(\mathcal{K}_{x}\right) & \leqslant 1+\operatorname{deg}_{Y} f(x, Y)+\sum_{y \in \mathbf{P}^{1}\left(\overline{\mathbf{F}}_{q}\right)} \operatorname{ord}_{y}(f(x, Y)) \\
& \leqslant 1+2 \operatorname{deg}_{Y} f(x, Y) \leqslant 2 \mathbf{c}(f),
\end{aligned}
$$

as claimed.

Remark 4.9. - Note that $\mathcal{K}_{x}$ is not always isomorphic to the ArtinSchreier sheaf $\mathcal{L}_{\psi\left(f_{x}\right)}$ on $\mathbf{A}^{1}$ : for instance, if $f=X / Y$ and $x=0$, we have $\mathcal{L}_{\psi(f(x, Y))}=\overline{\mathbf{Q}}_{\ell}$, but $\mathcal{K}_{0}=j_{!} \overline{\mathbf{Q}}_{\ell}$, where $j: \mathbf{A}^{1}-\{0\} \hookrightarrow \mathbf{A}^{1}$ is the open immersion. Thus $\mathcal{K}_{x}$ has zero stalk at 0 . However, this subtlety will not be a problem for us, in particular because the set of $x$ for which this behavior happens (and the set of $y$ such that the stalk of $\mathcal{K}_{x}$ at $y$ is not the same as that of $\left.\mathcal{L}_{\psi(f(x, Y))}\right)$ is finite and (since these points must be common zeros of the numerator $f_{1}$ and the denominator $f_{2}$ of $f$ ) has size bounded by $\operatorname{deg}\left(f_{1}\right) \operatorname{deg}\left(f_{2}\right)$, e.g. by Bézout's theorem.

In particular, we get the following corollary from the previous four lemmas. The statement uses the notation (4.6). 
Corollary 4.10. - Let $\mathbf{F}_{q}$ be a finite field of order $q$ and characteristic $p$, $\ell$ a prime distinct from $p$. Let $\mathcal{L}=\mathcal{L}_{\psi(f(X, Y))}$ be an $\ell$-adic Artin-Schreier sheaf on $\mathbf{A}^{2}$ over $\mathbf{F}_{q}$, where $f \in \mathbf{F}_{q}(X, Y)$ is a non-constant rational function. Let $\mathcal{F}$ be a middle-extension $\ell$-adic sheaf on $\mathbf{A}^{1}$ over $\mathbf{F}_{q}$.

For every $x \in \mathbf{A}^{1}\left(\overline{\mathbf{F}}_{q}\right)$, we have

$$
\operatorname{dim} H_{c}^{1}\left(\mathbf{A}^{1} \times \overline{\mathbf{F}}_{q}, \mathcal{F} \otimes \mathcal{L}_{x}\right) \leqslant 3 \cdot 2^{10} \mathbf{c}(f)^{4} \mathbf{c}(\mathcal{F})^{4} .
$$

Proof. - Combine Lemma 4.3, Lemma 4.4 and Lemma 4.8.

\subsection{A global bound for the conductor}

We now come to the lemma which contains the first idea in the proof of Theorem 2.3: it allows us to replace the sum of Swan conductors, in the definition of the conductor of a sheaf, by a global invariant (the EulerPoincare characteristic) that is more accessible to algebraic manipulations.

Lemma 4.11 (Global conductor bound). - Let $\mathcal{F}$ be a sheaf on $\mathbf{A}_{\mathbf{F}_{q}}^{1}$ without punctual sections. We have

$$
\mathbf{c}(\mathcal{F}) \leqslant 3 \operatorname{rank}(\mathcal{F})-\chi(\mathcal{F}) \leqslant 3 \operatorname{rank}(\mathcal{F})+h^{1}(\mathcal{F}) .
$$

Proof. - Let $U$ be the maximal open set on which $\mathcal{F}$ is lisse; it is dense. Since $\mathcal{F}$ has no punctual sections, we have

$$
\mathbf{c}(\mathcal{F})=\operatorname{rank}(\mathcal{F})+\sum_{x \in\left(\mathbf{P}^{1}-U\right)\left(\overline{\mathbf{F}}_{q}\right)}\left(1+\operatorname{Swan}_{x}(\mathcal{F})\right) .
$$

By the Euler-Poincaré formula (see, e.g., [9, 2.3.1]), we have

$$
-\chi_{c}(U, \mathcal{F})=-\operatorname{rank}(\mathcal{F}) \chi_{c}\left(\bar{U}, \overline{\mathbf{Q}}_{\ell}\right)+\sum_{x \in\left(\mathbf{P}^{1}-U\right)\left(\overline{\mathbf{F}}_{q}\right)} \operatorname{Swan}_{x}(\mathcal{F}) .
$$

We have $\chi_{c}\left(U, \overline{\mathbf{Q}}_{\ell}\right)=2-\left|\left(\mathbf{P}^{1}-U\right)\left(\overline{\mathbf{F}}_{q}\right)\right|=2-n(\mathcal{F})$, and therefore

$$
\sum_{x} \operatorname{Swan}_{x}(\mathcal{F})=-\chi_{c}(U, \mathcal{F})+(2-n(\mathcal{F})) \operatorname{rank}(\mathcal{F}) .
$$

By excision we have

$$
-\chi_{c}(U, \mathcal{F})=-\chi(\mathcal{F})+\sum_{x \in\left(\mathbf{A}^{1}-U\right)\left(\overline{\mathbf{F}}_{q}\right)} \operatorname{dim} \mathcal{F}_{x} \leqslant-\chi(\mathcal{F})+\sum_{x \in\left(\mathbf{P}^{1}-U\right)\left(\overline{\mathbf{F}}_{q}\right)} \operatorname{dim} \mathcal{F}_{x}
$$

and we obtain the upper bound

$$
\begin{aligned}
\mathbf{c}(\mathcal{F}) \leqslant 3 \operatorname{rank}(\mathcal{F})-\chi(\mathcal{F})+ & \sum_{x \in\left(\mathbf{P}^{1}-U\right)\left(\overline{\mathbf{F}}_{q}\right)}\left(1+\operatorname{dim} \mathcal{F}_{x}-\operatorname{rank}(\mathcal{F})\right) \\
& -220-
\end{aligned}
$$


Finally, since $\mathcal{F}$ is a middle extension sheaf, we have

$$
\operatorname{dim} \mathcal{F}_{x} \leqslant \operatorname{rank}(\mathcal{F})-1,
$$

for any $x \in\left(\mathbf{P}^{1}-U\right)\left(\overline{\mathbf{F}}_{q}\right)$, because $\mathcal{F}$ is not lisse at $x$. The first inequality follows, and also the second since

$$
-\chi(\mathcal{F}) \leqslant \operatorname{dim} H_{c}^{1}\left(\mathbf{A}_{\overline{\mathbf{F}}_{q}}^{1}, \mathcal{F}\right) .
$$

\section{Examples and applications}

\subsection{Preliminaries on trace functions}

The simplest applications of our results consist in plugging the trace functions of transform sheaves $T_{\mathcal{K}}(\mathcal{F})$ in any general result involving trace functions.

One must be slightly careful since many results are stated for irreducible middle-extension sheaves which are pointwise pure of some weight and the sheaf $T_{\mathcal{K}}(\mathcal{F})$ may not have these properties (in particular it may not be irreducible even if $\mathcal{F}$ is).

There is a potential notational subtlety (which did not arise in our previous works) involving the definition of weights. For an integer $n \in \mathbf{Z}$, recall (see [3, Def. 1.2.2]) that an $\ell$-adic sheaf $\mathcal{F}$ on $X_{\mathbf{F}_{q}}$ is pointwise pure of weight $n$ if, for all finite extensions $k / \mathbf{F}_{q}$ and for all $x \in X(k)$, the eigenvalues of Frobenius acting on $\mathcal{F}_{\bar{x}}$ are $|k|$-Weil numbers of some weight $w=n$. A sheaf $\mathcal{F}$ on $X$ is mixed of weights $\leqslant n$ if it has a finite filtration

$$
0=\mathcal{F}_{0} \subset \mathcal{F}_{1} \subset \cdots \subset \mathcal{F}_{m}=\mathcal{F}
$$

where the successive quotients $\mathcal{F}_{i} / \mathcal{F}_{i-1}$ are pointwise pure with weight $n_{i} \leqslant n$.

On the other hand (see $[10,(7.3 .7)]$ ), a middle-extension sheaf $\mathcal{F}$ on a curve $X_{\mathbf{F}_{q}}$ is pure of weight $n$ if, for some (equivalently any) dense open set $U \subset X$ where $\mathcal{F}$ is lisse, for all $k / \mathbf{F}_{q}$ and all $x \in U(k)$, the eigenvalues of Frobenius on $\mathcal{F}_{\bar{x}}$ are $|k|$-Weil numbers of weight $n$. It follows from results of Deligne (in particular [3, Lemme 1.8.1], and the Riemann Hypothesis) that such a sheaf is also mixed of weights $\leqslant n$, i.e., the eigenvalues of Frobenius at the "missing points" $X-U$ are also Weil numbers with weight $\leqslant n$. However, these weights may be $<n$. In other words, a middle-extension sheaf may be pure of weight $n$ without being pointwise pure of weight $n$.

The following lemma encapsulates a reduction of trace functions of constructible sheaves to middle-extension sheaves: 
Lemma 5.1 (Trace function of constructible sheaf). - Let $\mathbf{F}_{q}$ be a finite field of characteristic $p$, let $\ell \neq p$ be a prime and let $\mathcal{F}$ be an $\ell$-adic constructible sheaf on $\mathbf{A}_{\mathbf{F}_{q}}^{1}$ which is mixed of weights $\leqslant 0$.

There exists a decomposition of the trace function $t_{\mathcal{F}}$ of $\mathcal{F}$ of the form

$$
t_{\mathcal{F}}=t_{\mathcal{F}^{\text {mid }}}+t_{1}+t_{2},
$$

where $\mathcal{F}^{\text {mid }}$ is a middle-extension sheaf on $\mathbf{A}_{\mathbf{F}_{q}}^{1}$ which is pure of weight 0 , and where:

(1) The function $t_{1}$ is zero except for a set of values of $x \in \mathbf{F}_{q}$ of size at most $2 \mathbf{c}(\mathcal{F})$, and it satisfies

$$
\left|t_{1}(x)\right| \leqslant 2 \mathbf{c}(\mathcal{F})
$$

for all $x \in \mathbf{F}_{q}$.

(2) The function $t_{2}$ satisfies

$$
\left|t_{2}(x)\right| \leqslant \mathbf{c}(\mathcal{F}) q^{-1 / 2}
$$

for all $x \in \mathbf{F}_{q}$.

Proof. - This is a classical "dévissage". We begin by observing that

$$
\left|t_{\mathcal{F}}(x)\right| \leqslant \mathbf{c}(\mathcal{F})
$$

for all $x \in \mathbf{F}_{q}$ : indeed, by assumption, all eigenvalues of Frobenius on the stalk $\mathcal{F}_{\bar{x}}$ are of modulus at most 1 , and the maximal dimension of a stalk is bounded by the conductor (including where there is a punctual part of the sheaf).

Let $\mathcal{F}^{0}$ be the direct sum of quotients which are pointwise pure of weight 0 in a filtration of $\mathcal{F}$ with successive quotients which are pointwise pure of some weight $\leqslant 0$, and let $\mathcal{F}^{1}$ be the direct sum of the remaining quotients. We have

$$
t_{\mathcal{F}}(x)=t_{\mathcal{F}^{0}}(x)+t_{\mathcal{F}^{1}}(x),
$$

and trivially

$$
\left|t_{\mathcal{F}^{1}}(x)\right| \leqslant p^{-1 / 2} \mathbf{c}(\mathcal{F})
$$

for all $x \in \mathbf{F}_{q}$. We put $t_{2}=t_{\mathcal{F}^{1}}$.

Next, let

$$
0 \longrightarrow \mathcal{F}^{p c t} \longrightarrow \mathcal{F}^{0} \longrightarrow \mathcal{F}^{n p c t} \longrightarrow 0
$$

be the short exact sequence associated to the inclusion of the punctual part $\mathcal{F}^{p c t}$ of $\mathcal{F}^{0}$. We have

$$
t_{\mathcal{F}^{0}}(x)=t_{\mathcal{F}^{p c t}}(x)+t_{\mathcal{F}^{n p c t}}(x),
$$

and $t_{\mathcal{F} p c t}$ is zero except for $\leqslant \mathbf{c}(\mathcal{F})$ values of $x$ for which we have

$$
\left|t_{\mathcal{F}^{p c t}}(x)\right| \leqslant \operatorname{dim} H_{c}^{0}\left(\mathbf{A}^{1} \times \overline{\mathbf{F}}_{p}, \mathcal{F}^{0}\right) \leqslant \mathbf{c}(\mathcal{F}) .
$$


Finally, let $j: U \hookrightarrow \mathbf{A}^{1}$ be the open immersion of the maximal dense open subset where $\mathcal{F}^{n p c t}$ is lisse, and let

$$
\mathcal{F}^{\text {mid }}=j_{*} j^{*} \mathcal{F}^{\text {npct }} \text {. }
$$

This is a middle-extension sheaf, pointwise pure of weight 0 , with trace function equal to that of $\mathcal{F}$ for $x \in U\left(\mathbf{F}_{q}\right)$. Thus the difference

$$
t_{\mathcal{F}}-t_{\mathcal{F} \text { mid }}
$$

is zero except for at most $\mathbf{c}(\mathcal{F})$ values of $x \in \mathbf{F}_{q}$, and has modulus $\leqslant 2 \mathbf{c}(\mathcal{F})$ for all $x$. We obtain the desired decomposition by taking

$$
t_{1}=t_{\mathcal{F} \text { pct }}+t_{\mathcal{F}}-t_{\mathcal{F} \text { mid }} \text {. }
$$

We will apply the previous lemma to the trace functions of the transform sheaves $T_{\mathcal{K}}^{1}(\mathcal{F})$ considered in this paper. We introduce a definition for convenience.

Definition 5.2 ( $f$-disjoint sheaf). - Let $\mathbf{F}_{q}$ be a finite field of characteristic $p$, let $\ell \neq p$ be a prime. Let $f \in \mathbf{F}_{q}(X, Y)$ be a rational function and let $\mathcal{K}=\mathcal{L}_{\psi(f)}$ be the Artin-Schreier sheaf on $\mathbf{A}_{\mathbf{F}_{q}}^{2}$ associated to $f$.

A middle-extension sheaf $\mathcal{F}$ on $\mathbf{A}_{\mathbf{F}_{q}}^{1}$ is called $f$-disjoint or $\mathcal{K}$-disjoint if for all $x \in \mathbf{A}^{1}\left(\overline{\mathbf{F}}_{q}\right)$, one has

$$
H_{c}^{2}\left(\mathbf{A}^{1} \times \overline{\mathbf{F}}_{q}, \mathcal{F} \otimes \mathcal{K}_{x}\right)=0 .
$$

Corollary 5.3 (Artin-Schreier transforms as trace functions). - Let $\mathbf{F}_{q}$ be a finite field of characteristic $p$, let $\ell \neq p$ be a prime. Let $f \in \mathbf{F}_{q}(X, Y)$ be a rational function given by $f=f_{1} / f_{2}$ with $f_{i} \in \mathbf{F}_{q}[X, Y]$ coprime polynomials, and assume that $\mathbf{c}(f)<p$.

Let $\mathcal{F}$ be a middle-extension sheaf on $\mathbf{A}_{\mathbf{F}_{q}}^{1}$ which is pointwise pure of weight 0 and $f$-disjoint.

There exists an absolute constant $A \geqslant 1$, independent of $f$ and $\mathcal{F}$, such that for all $x \in \mathbf{A}^{1}\left(\mathbf{F}_{q}\right)$, we have

$$
\frac{1}{\sqrt{q}} \sum_{\substack{y \in \mathbf{F}_{q} \\ f_{2}(x, y) \neq 0}} t_{\mathcal{F}}(y) \psi(f(x, y))=-t_{0}(x)+t_{1}(x)+t_{2}(x),
$$

where

- $t_{0}$ is the trace function of a middle-extension sheaf $\mathcal{G}^{\text {mid }}$ of weight 0 on $\mathbf{A}_{\mathbf{F}_{q}}^{1}$ with

$$
\mathbf{c}\left(\mathcal{G}^{\text {mid }}\right) \leqslant(2 \mathbf{c}(f) \mathbf{c}(\mathcal{F}))^{A},
$$


- the function $t_{1}$ is zero for a set of values of $x \in \mathbf{F}_{q}$ of size at most $(2 \mathbf{c}(f) \mathbf{c}(\mathcal{F}))^{A}$, and it satisfies

$$
\left|t_{1}(x)\right| \leqslant(2 \mathbf{c}(f) \mathbf{c}(\mathcal{F}))^{A},
$$

for all $x \in \mathbf{F}_{q}$,

- The function $t_{2}$ satisfies

$$
\left|t_{2}(x)\right| \leqslant(2 \mathbf{c}(f) \mathbf{c}(\mathcal{F}))^{A} q^{-1 / 2}
$$

for all $x \in \mathbf{F}_{q}$.

Proof. - Let

$$
\mathcal{G}_{i}=T_{\mathcal{K}}^{i}(\mathcal{F})(1 / 2) \text { for } 0 \leqslant i \leqslant 2 \text { and } \mathcal{G}=\mathcal{G}_{1}=T_{\mathcal{K}}^{1}(\mathcal{F})(1 / 2) .
$$

By the Riemann Hypothesis [3] (taking into account the Tate twist) the sheaves $\mathcal{G}_{i}$ are mixed of weight $\leqslant i-1$ and in particular $\mathcal{G}$ is mixed of weight $\leqslant 0$. By the proper base change theorem (see Proposition 4.1(4)) and the Grothendieck-Lefschetz trace formula, the trace function of $\mathcal{G}$ is

$$
t_{\mathcal{G}}(x)=-\frac{1}{\sqrt{q}} \sum_{y \in \mathbf{F}_{q}} t_{\mathcal{F}}(y) t_{\mathcal{K}}(x, y)+t_{\mathcal{G}_{0}}(x)+t_{\mathcal{G}_{2}}(x)
$$

for $x \in \mathbf{F}_{q}$.

The stalk of $\mathcal{G}_{0}$ over $x$ is (by Lemma 4.2)

$$
H_{c}^{0}\left(\mathbf{A}^{1} \times \overline{\mathbf{F}}_{q}, \mathcal{F} \otimes \mathcal{L}_{x}\right)=0
$$

and that of $\mathcal{G}_{2}$ is (since $\mathcal{F}$ is $f$-disjoint)

$$
H_{c}^{2}\left(\mathbf{A}^{1} \times \overline{\mathbf{F}}_{q}, \mathcal{F} \otimes \mathcal{L}_{x}\right)=0 .
$$

Hence we obtain, for all $x \in \mathbf{F}_{q}$,

$$
t_{\mathcal{G}}(x)=-\frac{1}{\sqrt{q}} \sum_{y \in \mathbf{F}_{q}} t_{\mathcal{F}}(y) t_{\mathcal{K}}(x, y)
$$

By Definition 2.2, we have

$$
t_{\mathcal{K}}(x, y)= \begin{cases}\psi(f(x, y)) & \text { if } f_{2}(x, y) \neq 0 \\ 0 & \text { otherwise }\end{cases}
$$

and by Theorem 2.3 , there exists $A \geqslant 1$ such that the constructible sheaf $\mathcal{G}$ satisfies

$$
\mathbf{c}(\mathcal{G}) \leqslant(2 \mathbf{c}(f) \mathbf{c}(\mathcal{F}))^{A} .
$$

Thus the result follows by applying Lemma 5.1 to $\mathcal{G}$.

Definition 5.4. - The middle extension sheaf

$$
\mathcal{G}^{\text {mid }}=T_{\mathcal{K}}^{1}(\mathcal{F})^{\text {mid }}(1 / 2)
$$

obtained by applying Lemma 5.1 to $\mathcal{G}$ will be called the weight 0 part of $\mathcal{G}$. 
Remark 5.5. - For $\mathcal{K}$ as in this corollary, the condition that $\mathcal{F}$ is $f$ disjoint is valid in many cases. We list some of them for convenience.

The assumption of Corollary 5.3 holds:

(1) If $\mathcal{F}$ is irreducible of rank at least 2 (e.g., Kloosterman sheaves in one or more variables), or more generally if $\mathcal{F}$ is irreducible and not isomorphic to an Artin-Schreier sheaf.

(2) If $\mathcal{F}$ is tamely ramified and there is no specialization $f_{x}$ of $f$ which is constant as an element in $\mathbf{F}_{q}(Y)$ (e.g., any Kummer sheaf with trace function $\chi(g(x))$ for a multiplicative character $\chi$, provided no $f_{x}$ is constant); in particular, if $\mathcal{F}$ is the trivial sheaf with constant trace function 1 , it is enough that no specialization $f_{x}$ be constant.

(3) If $\mathcal{F}$ is an Artin-Schreier sheaf $\mathcal{L}_{\psi(g)}$ with trace function $\psi(g(x))$ and there is no $x \in \overline{\mathbf{F}}_{q}$ such that $g+f_{x}$ is constant.

\subsection{Application to automorphic twists}

We begin by explaining one setting where the application of our result is very easy:

Proposition 5.6. - Let $f$ be a Hecke cusp form of level $N \geqslant 1$ with Fourier coefficients $\varrho_{f}(n)$ at $\infty$. Let $g_{1}, g_{2} \in \mathbf{Z}[X, Y]$ be two non-constant coprime polynomials, and let $g=g_{1} / g_{2} \in \mathbf{Q}(X, Y)$.

Let $V$ be a smooth function on $] 0,+\infty[$ with compact support. Let $p$ be a prime number, let $K$ be an irreducible trace function modulo $p$ associated to a middle-extension sheaf $\mathcal{F}$ which is $(g(\bmod p))$-disjoint. For $\varepsilon>0$, we have

$$
\sum_{n \geqslant 1} \varrho_{f}(n) \frac{1}{\sqrt{p}}\left(\sum_{\substack{x \in \mathbf{F}_{p} \\ g_{2}(n, x) \neq 0(\bmod p)}} K(x) e\left(\frac{g_{1}(n, x) \overline{g_{2}(n, x)}}{p}\right)\right) V(n / p) \ll p^{1-1 / 8+\varepsilon}
$$

where the implied constant depends on $(f, V, \varepsilon, \mathbf{c}(\mathcal{F}), \mathbf{c}(g))$.

Proof. - The main result of [5] shows that

$$
\sum_{n \geqslant 1} \varrho_{f}(n) K(n) V(n / p) \ll p^{1-1 / 8+\varepsilon}
$$

if $K$ is the trace function of a geometrically isotypic middle-extension sheaf which is pointwise pure of weight 0 . We will show how to deduce the result from this. 
By Corollary 5.3 (applied with $\psi$ chosen so that $t_{\mathcal{L}_{\psi}}(x)=e(x / p)$ for $x \in \mathbf{F}_{p}$ ), we have a decomposition

$$
\frac{1}{\sqrt{p}}\left(\sum_{\substack{x \in \mathbf{F}_{p} \\ g_{2}(n, x) \neq 0}} K(x) e\left(\frac{g_{1}(n, x) \overline{g_{2}(n, x)}}{p}\right)\right)=-t_{0}(n)+t_{1}(n)+t_{2}(n)
$$

where $-t_{0}$ is the trace function of a middle-extension sheaf which is pure of weight 0 and has conductor $\leqslant C=(2 \mathbf{c}(f) \mathbf{c}(\mathcal{F}))^{A}$, while $t_{1}$ is zero except for $\leqslant C$ values of $x \in \mathbf{F}_{p}$, where it has modulus at most $C$, while $\left|t_{2}\right| \leqslant C p^{-1 / 2}$. We have then

$$
\sum_{n \geqslant 1} \varrho_{f}(n) t_{i}(n) V(n / p) \ll p^{1-1 / 8+\varepsilon}
$$

for $i=1,2$, and we are reduced to the case of $t_{0}$. Decomposing $t_{0}$ in trace functions of its geometrically isotypic components, we conclude by applying [5].

\subsection{Two-variable sums and the example of Conrey-Iwaniec}

A basic application of bounds on conductors like those of Theorem 2.3 concerns two-variable exponential sums of quite general type. We present the very general principle before giving a concrete example.

Given a trace function $K(x, y)$ in two variables, e.g.

$$
K(x, y)=\chi\left(f_{1}(x, y)\right) e\left(f_{2}(x, y) / p\right)
$$

for rational functions $f_{1}$ and $f_{2} \in \mathbf{F}_{p}(X, Y)$ and for a multiplicative character $\chi$ modulo $p$, one wishes to obtain square-root cancellation (when possible) for

$$
\sum_{x, y} K(x, y)
$$

This may be written as

$$
\sum_{x} \sum_{y} K(x, y)
$$

i.e., as the inner product of the constant function 1 (i.e., the trace function of the trivial sheaf) and (essentially) the trace function of

$$
T_{\mathcal{K}}^{1}\left(\overline{\mathbf{Q}}_{\ell}\right)
$$

where $\mathcal{K}$ is the sheaf with trace function $K$. It may happen that $K$ is given naturally as a product

$$
K(x, y)=K_{1}(x) K_{2}(y) K_{3}(x, y)
$$


for trace functions $K_{1}$ and $K_{2}$ modulo $p$ and another trace function $K_{3}$ in two variables; in such a case, it may be better to write the sum as

$$
\sum_{x} K_{1}(x) \sum_{y} K_{2}(y) K_{3}(x, y),
$$

which is the inner-product of $K_{1}$ with the trace function of $T_{\mathcal{K}_{3}}^{1}\left(\mathcal{K}_{2}\right)$, with obvious notation.

From a direct application of the Riemann Hypothesis, we obtain the following qualitative information concerning these types of sums:

Proposition 5.7 (Small diagonal principle). - Let $\mathbf{F}_{q}$ be a finite field of characteristic $p$, let $\ell \neq p$ be a prime number. Let $\mathcal{K}$ be a constructible $\ell$-adic sheaf mixed of weight $\leqslant 0$ on $\mathbf{A}_{\mathbf{F}_{q}}^{2}$.

Let $\mathcal{F}_{2}$ be a middle-extension sheaf on $\mathbf{A}_{\mathbf{F}_{q}}^{1}$, pointwise pure of weight 0 such that $T_{\mathcal{K}}^{2}\left(\mathcal{F}_{2}\right)$ is generically 0 .

There exists a finite set $X\left(\mathcal{K}, \mathcal{F}_{2}\right)$ of geometrically irreducible middleextension sheaves which are pointwise pure of weight 0 , of cardinality bounded in terms of the conductor of $T_{\mathcal{K}}^{1}\left(\mathcal{F}_{2}\right)$, such that if $\mathcal{F}_{1}$ is a middle-extension sheaf of weight 0 , geometrically irreducible, and not geometrically isomorphic to any of the sheaves in $X\left(\mathcal{K}, \mathcal{F}_{2}\right)$, then

$$
\sum_{x, y \in \mathbf{F}_{q}} t_{\mathcal{F}_{1}}(x) t_{\mathcal{F}_{2}}(y) t_{\mathcal{K}}(x, y) \ll q,
$$

where the implied constant depends only on the conductor of $\mathcal{F}_{1}$ and of $T_{\mathcal{K}}^{1}\left(\mathcal{F}_{2}\right)$.

Proof. - Let $X\left(\mathcal{K}, \mathcal{F}_{2}\right)$ be the set of geometric isomorphism classes of geometrically irreducible components of the weight 0 part of $\mathcal{G}=T_{\mathcal{K}}^{1}\left(\mathcal{F}_{2}\right)(1 / 2)$. This is a finite set of cardinality bounded by the rank of $T_{\mathcal{K}}^{1}\left(\mathcal{F}_{2}\right)$, hence bounded in terms of the conductor of $\mathcal{F}_{2}$.

Under the assumptions of the proposition, for $\mathcal{F}_{1}$ geometrically irreducible and not in $X\left(\mathcal{K}, \mathcal{F}_{2}\right)$, we have

$$
\begin{aligned}
\frac{1}{q^{1 / 2}} \sum_{x, y \in \mathbf{F}_{q}} t_{\mathcal{F}_{1}}(x) t_{\mathcal{F}_{2}}(y) t_{\mathcal{K}}(x, y) & =\sum_{x \in \mathbf{F}_{q}} t_{\mathcal{F}_{1}}(x) \frac{1}{q^{1 / 2}} \sum_{y \in \mathbf{F}_{q}} t_{\mathcal{F}_{2}}(y) t_{\mathcal{K}}(x, y) \\
& =-\operatorname{tr}\left(\operatorname{Fr} \mid H_{c}^{1}\left(\mathbf{A}^{1} \times \overline{\mathbf{F}}_{q}, \mathcal{F}_{1} \otimes \mathcal{G}\right)\right)
\end{aligned}
$$

(since the cohomology spaces $H_{c}^{0}$ and $H_{c}^{2}$ vanishing here). The first cohomology space is mixed of weights $\leqslant 1$ by Deligne's Riemann Hypothesis, and hence we obtain the result using the conductor bounds (Lemmas 4.3 and 4.4). 
Although this proposition does not, by itself, give square-root cancellation in any individual case, it implies for instance that

$$
\sum_{x} e\left(\frac{a x^{2}}{p}\right) \sum_{y} t_{\mathcal{F}_{2}}(y) t_{\mathcal{K}}(x, y) \ll p
$$

(working over $\mathbf{F}_{p}$ ) for all $a \in \mathbf{F}_{p}$ except for a number of exceptions bounded in terms of the conductors of $\mathcal{F}_{2}$ and $\mathcal{K}$ only. In quite a few applications, this type of qualitative "control of the diagonal" is sufficient (for instance, similar ideas are crucial in [5]). However, this is not always the case, and one needs to attempt some further analysis if a more precise result is needed.

We now present a concrete example, taken from the important work of Conrey and Iwaniec on the third moment of special values of automorphic $L$-functions [1]. Given a prime $p$ and two multiplicative characters $\chi_{1}$ and $\chi_{2}$ modulo $p$, Conrey and Iwaniec consider the sum

$$
\begin{aligned}
S\left(\chi_{1}, \chi_{2}\right) & =\sum_{x, y \in \mathbf{F}_{p}} \chi_{1}(x y(x+1)(y+1)) \chi_{2}(x y-1) \\
& =\sum_{x \in \mathbf{F}_{p}} \chi_{1}(x(x+1)) \sum_{x, y \in \mathbf{F}_{p}} \chi_{1}(y(y+1)) \chi_{2}(x y-1) .
\end{aligned}
$$

They prove:

THEOREM 5.8 (Conrey-Iwaniec). - Let $\chi_{1}$ be a non-trivial multiplicative character modulo $p$, and let $\chi_{2}$ be any multiplicative character modulo p. Then

$$
S\left(\chi_{1}, \chi_{2}\right) \ll p
$$

where the implied constant is absolute.

This is [1, Lemma 13.1], slightly generalized, since we do not assume that $\chi_{1}$ is a real character. Conrey and Iwaniec remark [1, Remarks, p. 1208] that their main result concerning $L$-functions would be considerably weakened if (for $\chi_{1}$ a real character modulo $p$, for many primes $p$ ) there existed a single character $\chi_{2}$ for which the size of the sum would be $p^{3 / 2}$.

Remark 5.9. - Some natural generalizations of these sums have appeared recently in the work of Petrow and Young [19] on the Weyl bound for Dirichlet $L$-functions. They prove the analogue of the theorem of Conrey and Iwaniec using some of the ideas in this paper.

We will explain how to prove Theorem 5.8 using the ideas of cohomological transforms. The sums $p^{-1 / 2} S\left(\chi_{1}, \chi_{2}\right)$ are naturally presented in the form discussed above, namely as the inner product of the trace function of the dual of the Kummer sheaf

$$
\begin{aligned}
\mathcal{F}_{1}= & \mathcal{L}_{\chi_{1}(X(X+1))} \\
& -228-
\end{aligned}
$$


with that of the transform sheaf

$$
\mathcal{G}=T_{\mathcal{K}}^{1}\left(\mathcal{F}_{1}\right)(1 / 2)=T_{\mathcal{K}}^{1}\left(\mathcal{L}_{\chi_{1}(Y(Y+1))}\right)(1 / 2) \quad \text { where } \mathcal{K}=\mathcal{L}_{\chi_{2}(X Y-1)}
$$

(the latter is defined as the extension by 0 of the Kummer sheaf $\mathcal{L}_{\chi_{2}(X Y-1)}$ on the open set complement of the curve $X Y-1$, see below for the general definition).

More precisely, the trace function of $\mathcal{G}$ is

$$
t_{\mathcal{G}}(x)=-\frac{1}{p^{1 / 2}} \sum_{y \in \mathbf{F}_{p}} \chi_{1}(y(y+1)) \chi_{2}(x y-1)
$$

for all $x \in \mathbf{F}_{p}$, provided $\chi_{1} \neq 1$ : indeed, by the trace formula and the proper base change theorem, it is enough to show that $T_{\mathcal{K}}^{0}\left(\mathcal{L}_{\chi_{1}(Y(Y+1))}\right)=$ $T_{\mathcal{K}}^{2}\left(\mathcal{L}_{\chi_{1}(Y(Y+1))}\right)=0$ in that case. The former is true by Lemma 4.2 , and the latter because the fiber above $x \in \overline{\mathbf{F}}_{p}$ is

$$
H_{c}^{2}\left(\mathbf{A}^{1} \times \overline{\mathbf{F}}_{p}, \mathcal{L}_{\chi_{1}(Y(Y+1))} \otimes \mathcal{L}_{\chi_{2}(x Y-1)}\right)=0
$$

(since $\chi_{1} \neq 1$, this can only be non-zero if the second tensor factor is ramified at 0 and -1 , but it is in fact always unramified at 0 ).

The kernel $\mathcal{K}$ is not of the type considered in Theorem 2.3. However, it is easy to adapt the proof of this result to derive an analogue for multiplicative characters. These we define in general in analogy with Definition 2.2:

DEFINITION 5.10. - Given a non-trivial multiplicative $\ell$-adic character of $\mathbf{F}_{q}^{\times}$, we denote by $\mathcal{L}_{\chi}$ the associated Kummer sheaf on $\mathbf{G}_{m, \mathbf{F}_{q}}$ (see [2, Sommes Trig.]). Let $f \in \mathbf{F}_{q}(X, Y)$ be a rational function, $U$ the open set where the numerator and denominator are both non-zero, with $j: U \hookrightarrow \mathbf{A}^{2}$ the open immersion; let $f_{U}: U \rightarrow \mathbf{G}_{m}$ be the associated morphism, then the Kummer sheaf associated to $f$ is the constructible $\ell$-adic sheaf on $\mathbf{A}_{\mathbf{F}_{q}}^{2}$ defined as

$$
\mathcal{L}_{\chi(f)}=j_{!} f_{U}^{*} \mathcal{L}_{\chi}
$$

TheOREM 5.11 (Conductor of Kummer transforms). - Let $\mathbf{F}_{q}$ be a finite field of order $q$ and characteristic $p, \ell$ a prime distinct from $p$. Let $\mathcal{K}$ be an $\ell$-adic sheaf on $\mathbf{A}^{1} \times \mathbf{A}^{1}$ over $\mathbf{F}_{q}$ of the form $\mathcal{K}=\mathcal{L}_{\chi(f)}$.

For constructible sheaves $\mathcal{F}$ on $\mathbf{A}_{\mathbf{F}_{q}}^{1}$, and $0 \leqslant i \leqslant 2$, let

$$
T_{\mathcal{K}}^{i}(\mathcal{F})=R^{i} p_{1, !}\left(p_{2}^{*} \mathcal{F} \otimes \mathcal{K}\right) .
$$

The sheaves $T_{\mathcal{K}}^{i}(\mathcal{F})$ are constructible and there exists an absolute constant $A \geqslant 1$ such that

$$
\mathbf{c}\left(T_{\mathcal{K}}^{i}(\mathcal{F})\right) \leqslant(2 \mathbf{c}(\mathcal{K}) \mathbf{c}(\mathcal{F}))^{A}
$$

and moreover

$$
\operatorname{dim} H_{c}^{i}\left(\mathbf{A}^{2} \times \overline{\mathbf{F}}_{q}, p_{2}^{*} \mathcal{F} \otimes \mathcal{K}\right) \leqslant(2 \mathbf{c}(f) \mathbf{c}(\mathcal{F}))^{A} .
$$


Sketch of proof. - One can follow line by line the proof of Theorems 2.3 and 2.5. The only differences are:

(1) we deal separately with the case

$$
f(X, Y)=f_{1}(X) f_{2}(Y)
$$

(instead of $f(X, Y)=f_{1}(X)+f_{2}(Y)$ in the case of an additive character);

(2) to bound the Betti numbers

$$
\operatorname{dim} H_{c}^{i}\left(\mathbf{A}^{2} \times \overline{\mathbf{F}}_{q}, \mathcal{K}\right)
$$

(i.e., when the input sheaf is trivial), one uses the results of Adolphson-Sperber or Katz [14, Thm. 12] instead of those of Bombieri (which are only proved for additive characters); an alternative is to lift the tame sheaves to characteristic 0 .

In particular, in our case, $\mathcal{G}=T_{\mathcal{K}}^{1}\left(\mathcal{L}_{\chi_{1}(Y(Y+1))}\right)(1 / 2)$ has conductor absolutely bounded as $\chi_{1}, \chi_{2}$ and $p$ vary. By the Riemann Hypothesis, the sheaf $\mathcal{G}$ is also mixed of weights $\leqslant 0$, and therefore the principle above shows that, for all primes $p$, and for all characters $\chi_{2}$, we have

$$
S\left(\chi_{1}, \chi_{2}\right) \ll p
$$

with an absolute implied constant, for all but a bounded number of multiplicative characters $\chi_{1}$ modulo $p$ (since $\mathcal{L}_{\chi_{1}(X(X+1))} \simeq \mathcal{L}_{\chi_{1}^{\prime}(X(X+1))}$ if and only if $\left.\chi_{1}=\chi_{1}^{\prime}\right)$.

In order to go deeper and show that, in fact, these exceptions do not exist, we must look more carefully at $\mathcal{G}$.

Proposition 5.12. - Let $\chi_{1}$ and $\chi_{2}$ be non-trivial characters of $\mathbf{F}_{q}^{\times}$, and let $\mathcal{F}_{1}, \mathcal{K}$ and $\mathcal{G}$ be the constructible sheaves defined in (5.2) and (5.3) and let $\mathcal{G}^{0}$ be the weight 0 part of $\mathcal{G}$.

(1) The sheaves $\mathcal{G}$ and $\mathcal{G}^{0}$ have generic rank 2 .

(2) The sheaf $\mathcal{G}^{0}$ is lisse on $W=\mathbf{A}^{1}-\{0,-1\}$ and geometrically irreducible.

If we grant this proposition let us show how to conclude the proof of Theorem 5.8. By Lemma 5.1, we have

$$
p^{-1 / 2} S\left(\chi_{1}, \chi_{2}\right)=\sum_{x \in \mathbf{F}_{p}} t_{\mathcal{F}_{1}}(x) t_{\mathcal{G}}(x)=\sum_{x \in \mathbf{F}_{p}} t_{\mathcal{F}_{1}}(x) t_{\mathcal{G}^{0}}(x)+O\left(p^{1 / 2}\right)
$$

where the implicit constant is absolute. Since $\mathcal{G}^{0}$ is geometrically irreducible of rank 2 on $W$ and $\mathcal{F}_{1}$ has rank 1 , the sheaf $\mathcal{G}^{0}$ cannot not be geometrically 
isomorphic to the dual of $\mathcal{F}_{1}$, and hence

$$
p^{-1 / 2} S\left(\chi_{1}, \chi_{2}\right)=O\left(p^{1 / 2}\right)
$$

where the implied constant is absolute, by the Riemann Hypothesis and the conductor bound $\mathbf{c}\left(\mathcal{G}^{0}\right) \ll 1$. This finishes the proof of Theorem 5.8.

For the proof of (2), we recall a very useful diophantine criterion for irreducibility of Katz (see [12, Lemma 7.0.3]).

Lemma 5.13 (Irreducibility criterion). - Let $\mathbf{F}_{q}$ be a finite field of characteristic $p$, let $\ell \neq p$ be a prime number and let $\mathcal{F}$ be an $\ell$-adic constructible sheaf on $\mathbf{A}_{\mathbf{F}_{p}}^{1}$ which is mixed of weights $\leqslant 0$. Then we have

$$
\frac{1}{q^{\nu}} \sum_{x \in \mathbf{F}_{q^{\nu}}}\left|t_{\mathcal{F}}\left(x, q^{\nu}\right)\right|^{2}=1+O\left(q^{-\nu / 2}\right)
$$

for $\nu \geqslant 1$, if and only if the middle-extension part of weight 0 of $\mathcal{F}$ is geometrically irreducible, i.e., if and only if, for any dense open subset $U$ where $\mathcal{F}$ is lisse, the restriction of the weight 0 part of $\mathcal{F}$ to $U \times \overline{\mathbf{F}}_{q}$ corresponds to an irreducible representation of the geometric fundamental group of $U$.

Proof. - For $\nu \geqslant 1$ fixed, let

$$
t_{\mathcal{F}}\left(x, q^{\nu}\right)=t_{\mathcal{F}^{\text {mid }}}\left(x, q^{\nu}\right)+t_{1}(x)+t_{2}(x)
$$

for $x \in \mathbf{F}_{q^{\nu}}$ be the decomposition of Lemma 5.1 (applied to $\mathbf{F}_{q^{\nu}}$ ). We wish to prove that $\mathcal{F}^{\text {mid }}$ is geometrically irreducible. From the properties of $t_{1}$ and $t_{2}$, we see that

$$
\frac{1}{q^{\nu}} \sum_{x \in \mathbf{F}_{q^{\nu}}}\left|t_{\mathcal{F}}\left(x, q^{\nu}\right)\right|^{2}=\frac{1}{q^{\nu}} \sum_{x \in \mathbf{F}_{q^{\nu}}}\left|t_{\mathcal{F}^{m i d}}\left(x, q^{\nu}\right)\right|^{2}+O\left(q^{-\nu}\right)
$$

for $\nu \geqslant 1$. Now let $U$ be a dense open subset of $\mathbf{A}^{1}$ where $\mathcal{F}^{\text {mid }}$ is lisse. Then we have

$$
\frac{1}{q^{\nu}} \sum_{x \in U\left(\mathbf{F}_{q^{\nu}}\right)}\left|t_{\mathcal{F}^{m i d}}\left(x, q^{\nu}\right)\right|^{2}=\frac{1}{q^{\nu}} \sum_{x \in \mathbf{F}_{q^{\nu}}}\left|t_{\mathcal{F}^{m i d}}\left(x, q^{\nu}\right)\right|^{2}+O\left(q^{-\nu}\right),
$$

for $\nu \geqslant 1$, since the complement is finite. Therefore, we have (5.4) if and only if

$$
\frac{1}{q^{\nu}} \sum_{x \in U\left(\mathbf{F}_{q^{\nu}}\right)}\left|t_{\mathcal{F}^{\text {mid }}}\left(x, q^{\nu}\right)\right|^{2}=1+O\left(q^{-\nu / 2}\right)
$$

for $\nu \geqslant 1$. But by [12, Lemma 7.0.3] applied to the lisse sheaf $\mathcal{F}^{\text {mid }}$ on $U$, which is pure of weight 0 , this last condition holds if and only if $\mathcal{F}^{\text {mid }}$ is geometrically irreducible on $U$. 
Proof of Proposition 5.12. - We begin by checking the generic rank of $\mathcal{G}$. The fiber of $\mathcal{G}$ over $x \in \overline{\mathbf{F}}_{q}$ is

$$
H_{c}^{1}\left(\mathbf{A}^{1} \times \overline{\mathbf{F}}_{q}, \mathcal{L}_{\chi_{1}(Y(Y+1))} \otimes \mathcal{L}_{\chi_{2}(x Y-1)}\right)(1 / 2) .
$$

By the Euler-Poincaré formula (see (4.5)), its dimension is

$$
\operatorname{dim} H_{c}^{1}\left(\mathbf{A}^{1} \times \overline{\mathbf{F}}_{q}, \mathcal{L}_{\chi_{1}(Y(Y+1))} \otimes \mathcal{L}_{\chi_{2}(x Y-1)}\right)=-1+3=2
$$

if $x \neq-1$ (so that the sheaf is ramified at the three points $y=0,-1$ and $1 / x)$. Hence the generic rank of $\mathcal{G}$ is 2 .

We next apply the irreducibility criterion to $\mathcal{G}$, which is mixed of weights $\leqslant 0$, to prove that the part of weight 0 is geometrically irreducible on any dense open set where it is lisse.

For $\nu \geqslant 1$ and $i=1,2$, we denote by $\chi_{i, \nu}$ the extension $\chi_{i} \circ N_{\mathbf{F}_{q^{\nu}} / \mathbf{F}_{q}}$ of $\chi_{i}$ to $\mathbf{F}_{q^{\nu}}$, we have

$$
\begin{aligned}
\frac{1}{q^{\nu}} \sum_{x \in \mathbf{F}_{q^{\nu}}}\left|t_{\mathcal{G}}\left(x, q^{\nu}\right)\right|^{2} & =\frac{1}{q^{2 \nu}} \sum_{x \in \mathbf{F}_{q^{\nu}}}\left|\sum_{y \in \mathbf{F}_{q^{\nu}}} t_{\mathcal{F}_{1}}\left(x, q^{\nu}\right) \chi_{2, \nu}(x y-1)\right|^{2} \\
= & \frac{1}{q^{2 \nu}} \sum_{y_{1}, y_{2} \in \mathbf{F}_{q^{\nu}}} t_{\mathcal{F}_{1}}\left(y_{1}, q^{\nu}\right) \overline{t_{\mathcal{F}_{1}}\left(y_{2}, q^{\nu}\right)} \\
& \times \sum_{x \in \mathbf{F}_{q^{\nu}}} \chi_{2, \nu}\left(x y_{1}-1\right) \overline{\chi_{2, \nu}\left(x y_{2}-1\right)}
\end{aligned}
$$

The contribution of the diagonal terms $y_{1}=y_{2}$ to this sum is

$$
\begin{aligned}
\frac{q^{\nu}-1}{q^{2 \nu}} \sum_{y \in \mathbf{F}_{q^{\nu}}}\left|t_{\mathcal{F}_{1}}\left(y, q^{\nu}\right)\right|^{2} & =\frac{1}{q^{\nu}} \sum_{y \in \mathbf{F}_{q^{\nu}}}\left|t_{\mathcal{F}_{1}}\left(y, q^{\nu}\right)\right|^{2}+O\left(q^{-\nu}\right) \\
& =1+O\left(q^{-\nu}\right)
\end{aligned}
$$

since $t_{\mathcal{F}_{1}}\left(y, q^{\nu}\right)=\chi_{1, \nu}(y(y+1))$.

If $y_{1} \neq y_{2}$, the map

$$
x \longmapsto \frac{x y_{1}-1}{x y_{2}-1}
$$

is a bijection on $\mathbf{P}^{1}\left(\mathbf{F}_{q^{\nu}}\right)$. Hence, in that case, we have

$$
\sum_{x \in \mathbf{F}_{q^{\nu}}} \chi_{2, \nu}\left(x y_{1}-1\right) \overline{\chi_{2, \nu}\left(x y_{2}-1\right)}=-\chi_{2, \nu}\left(y_{1}\right) \overline{\chi_{2, \nu}\left(y_{2}\right)}
$$

(we write it in this way to incorporate the case $y_{2}=0$, in which case the map is a bijection of $\mathbf{F}_{q^{\nu}}$, while otherwise the sum over $x \in \mathbf{F}_{q^{\nu}}$ misses the point $\left.y_{1} / y_{2}\right)$. 
Thus we get an off-diagonal contribution equal to

$$
-\frac{1}{q^{2 \nu}} \sum_{\substack{y_{1}, y_{2} \in \mathbf{F}_{q^{\nu}} \\ y_{1} \neq y_{2}}} t_{\mathcal{F}_{1}}\left(y_{1}, q^{\nu}\right) \overline{t_{\mathcal{F}_{1}}\left(y_{2}, q^{\nu}\right)} \chi_{2, \nu}\left(y_{1}\right) \overline{\chi_{2, \nu}\left(y_{2}\right)} .
$$

Inserting the diagonal in this sum, we find that it is equal to

$$
-\frac{1}{q^{2 \nu}}\left(\left|\sum_{y \in \mathbf{F}_{q^{\nu}}} t_{\mathcal{F}_{1}}\left(y, q^{\nu}\right) \chi_{2, \nu}(y)\right|^{2}-\sum_{y \in \mathbf{F}_{q^{\nu}}^{\times}}\left|t_{\mathcal{F}_{1}}\left(y, q^{\nu}\right)\right|^{2}\right) .
$$

Since $\mathcal{F}_{1}$ is geometrically irreducible but not geometrically isomorphic to $\mathcal{L}_{\chi_{2}}$ (indeed $\mathcal{F}_{1}$ is ramified at -1 while $\mathcal{L}_{\chi_{2}}$ is lisse there), by the Riemann Hypothesis (in that case, due to A.Weil), we have

$$
\left|\sum_{y \in \mathbf{F}_{q^{\nu}}} t_{\mathcal{F}_{1}}\left(y, q^{\nu}\right) \chi_{2, \nu}(y)\right|^{2}=O\left(q^{\nu}\right)
$$

while the bound

$$
\sum_{y \in \mathbf{F}_{q^{\nu}}^{\times}}\left|t_{\mathcal{F}_{1}}\left(y, q^{\nu}\right)\right|^{2}=O\left(q^{\nu}\right)
$$

is immediate. Hence the off-diagonal contribution is $O\left(q^{-\nu}\right)$, and the irreducibility criterion does apply.

Thus $\mathcal{G}^{0}$ is geometrically irreducible on any open set where it is lisse. We will now prove that $\mathcal{G}$ is lisse and pure of weight 0 on $W$. It then follows that $\mathcal{G}=\mathcal{G}^{0}$ on $W$, which will conclude the proof of the proposition.

We begin by checking that $\mathcal{G}$ is lisse on $W=\mathbf{A}^{1}-\{0,-1\}$ using Deligne's semicontinuity theorem ([16, Cor. 2.1.2]). We denote by $p_{1}$ and $p_{2}$ the projections $(x, y) \mapsto x$ and $(x, y) \mapsto y$ from $\mathbf{A}^{2}$ to $\mathbf{A}^{1}$. We also denote by $\widetilde{p}_{1}: \mathbf{A}^{1} \times \mathbf{P}^{1} \rightarrow \mathbf{A}^{1}$ the first projection. This is a smooth and proper morphism of relative dimension 1 . Let $\mathcal{H}=\mathcal{L}_{\chi_{2}(X Y-1)} \otimes p_{2}^{*} \mathcal{L}_{\chi_{1}(Y(Y+1))}$ so that $\mathcal{G}=R^{1} p_{1, !} \mathcal{H}(1 / 2)$. Note that $\mathcal{H}$ is lisse on $U=\mathbf{A}^{2}-D$ where $D$ is the divisor

$$
D=\{X Y=1\} \cup\left(\mathbf{A}^{1} \times\{0\}\right) \cup\left(\mathbf{A}^{1} \times\{-1\}\right) .
$$

We denote by $\widetilde{\mathcal{H}}$ the sheaf on $\mathbf{A}^{1} \times \mathbf{P}^{1}$ which is the extension by zero of $\widetilde{\mathcal{H}}$ from $\mathbf{A}^{1} \times \mathbf{A}^{1}$ to $\mathbf{A}^{1} \times \mathbf{P}^{1}$. By definition, we have $\mathcal{G}=R^{1} \widetilde{p}_{1, *} \widetilde{\mathcal{H}}(1 / 2)$.

Let $\widetilde{D}$ be the complement in $\mathbf{A}^{1} \times \mathbf{P}^{1}$ of the open set $U$. This is the union of $D$ and of the line $\mathbf{A}^{1} \times\{\infty\}$.

Let $X=\widetilde{p}_{1}^{-1}(W)$. By restriction, the morphism $\widetilde{p}_{1}$ defines a proper smooth morphism $X \rightarrow W$ of relative dimension 1 . The intersection $\widetilde{D} \cap X$ 
is a divisor in $X$ that is flat and finite (of degree 4 ) over $W$. The sheaf $\widetilde{\mathcal{H}}$ is lisse on the complement of $\widetilde{D} \cap X$ in $\mathbf{A}^{1} \times \mathbf{P}^{1}$.

Let $x \in W$. The fiber $C_{x}$ of $\widetilde{p}_{1}$ over $x$ is identified with $\mathbf{P}^{1}$, and the restriction of $\widetilde{\mathcal{H}}$ to $C_{x}$ is identified with a lisse sheaf on the dense open set

$$
U_{x}=\mathbf{A}^{1}-\{0,-1,1 / x, \infty\} \subset \mathbf{P}^{1} .
$$

The restriction of the sheaf $\widetilde{\mathcal{H}}$ to $C_{x}$ is (at most) tamely ramified everywhere, hence the function $\varphi$ of [16, Thm. 2.1.1] is constant equal to 0 on points of $W$. Corollary 2.1.2 of loc. cit. then implies that $\mathcal{G}$ is lisse on $W$, as claimed.

We finally prove that $\mathcal{G}$ is pure of weight 1 on $W$. We apply $[15$, Lemma $4.22(\mathrm{~b})]$ to the morphism $\widetilde{p}_{1}: X \rightarrow W$ and to the sheaf $\widetilde{\mathcal{H}}$ on $X$. For any $x \in \mathbf{P}^{1}$, the sheaf $\widetilde{\mathcal{H}}_{x}$, after pullback to $C_{x}=\{x\} \times \mathbf{P}^{1}$, has neither punctual section nor trivial subrepresentation (as lisse sheaf on $U_{x}$ ). Thus the assumptions of loc. cit. are satisfied. It follows that for any $x \in W$, the part of weight $<1$ of the stalk at $x$ of $\mathcal{G}^{\text {mid }}$ is isomorphic to

$$
\bigoplus_{y \in C_{x}-U_{x}}\left(\widetilde{\mathcal{H}}_{x}\right)_{\bar{\eta}}^{I_{y}} /\left(\widetilde{\mathcal{H}}_{x}\right)_{\bar{y}}
$$

But already $\left(\widetilde{\mathcal{H}}_{x}\right)_{\bar{\eta}}^{I_{y}}=0$ at all singular points $y \in\{0,-1,1 / x, \infty\}$, so this direct sum vanishes.

Remark 5.14. - The irreducibility criterion applies more generally to show that $T_{\mathcal{K}}(\mathcal{F})^{\text {mid }}$ is geometrically irreducible as long as $\mathcal{F}$ is a geometrically irreducible middle-extension sheaf, pure of weight 0 , which is not geometrically isomorphic to $\mathcal{L}_{\chi_{2}}$. Indeed, under these assumptions, the irreducibility criterion shows that (5.5) holds with $\mathcal{F}_{1}$ replaced by $\mathcal{F}$, while (5.6) follows from the Riemann Hypothesis of Deligne.

\section{Setting up the proof}

To clarify the proof of Theorems 2.3 and 2.5, and in view of further generalizations, we introduce the following definition:

Definition 6.1 (Continuity).

(1) Let

$$
i:(f, \mathcal{F}) \longmapsto i(f, \mathcal{F})
$$

be any real-valued map taking a pair $(f, \mathcal{F})$ as input, where $f$ is a non-constant rational function in $\mathbf{F}_{q}(X, Y)$ for some finite field $\mathbf{F}_{q}$ and $\mathcal{F}$ is a middle-extension $\ell$-adic sheaf on the affine line over $\mathbf{F}_{q}$. 
Then we say that $i$ is continuous if there exists an integer $C \geqslant 1$ such that

$$
|i(f, \mathcal{F})| \leqslant(2 \mathbf{c}(f) \mathbf{c}(\mathcal{F}))^{C}
$$

for all pairs $(f, \mathcal{F})$ as above such that $\mathbf{c}(f)<p . .^{(1)}$

(2) Similarly, if

$$
j: f \longmapsto j(f)(\text { resp. } k: \mathcal{F} \longmapsto k(\mathcal{F}))
$$

are real-valued maps taking as input a non-constant rational function $f \in \mathbf{F}_{q}(X, Y)$ for some finite field $\mathbf{F}_{q}$ (resp. a middle-extension $\ell$-adic sheaf $\mathcal{F}$ on the affine line over $\mathbf{F}_{q}$ ), then we say that $j$ (resp. $k$ ) is continuous if and only if there exists an integer $C \geqslant 1$ such that

$$
|j(f)| \leqslant(2 \mathbf{c}(f))^{C}\left(\operatorname{resp} .|k(\mathcal{F})| \leqslant(2 \mathbf{c}(\mathcal{F}))^{C}\right),
$$

for all $f$ with $\mathbf{c}(f)<p$ (resp. all middle-extension sheaves $\mathcal{F})$.

Remark 6.2. - Some of our arguments are easier to follow and check if one uses a weaker definition of continuity, where one only asks that

$$
|i(f, \mathcal{F})| \leqslant \Psi(\mathbf{c}(f), \mathbf{c}(\mathcal{F}))
$$

for some function $\Psi$ taking positive integral values. For some basic applications, such a statement is also sufficient, and the reader might wish to consider this as the notion of continuity in a first reading.

Example 6.3. - For instance, Theorem 2.3 asserts that the maps

$$
(f, \mathcal{F}) \longmapsto \mathbf{c}\left(T_{\mathcal{K}}^{i}(\mathcal{F})\right)
$$

are continuous, and Theorem 2.5 that the maps

$$
(f, \mathcal{F}) \longmapsto \operatorname{dim} H_{c}^{i}\left(\mathbf{A}^{2} \times \overline{\mathbf{F}}_{q}, p_{2}^{*} \mathcal{F} \otimes \mathcal{K}\right)
$$

are continuous. Lemma 4.3 proves that the functions

$$
\mathcal{F} \longmapsto \operatorname{dim} H_{c}^{i}\left(\mathbf{A}^{1} \times \overline{\mathbf{F}}_{q}, \mathcal{F}\right)
$$

are continuous.

Clearly, if we fix one argument of a continuous map $i(f, \mathcal{F})$ and let the other vary, this gives a continuous map of this second argument. Also, a sum $i_{1}+i_{2}$ of continuous functions is also continuous, as well as a product $i_{1} i_{2}$.

(1) This restriction on $\mathbf{c}(\mathcal{F})$ may seem artificial, and it is possible that it would not be needed for our results. But it has no influence on the applications. 
For simplicity, we denote

$$
\begin{aligned}
c_{i}(f, \mathcal{F}) & =\mathbf{c}\left(T_{\mathcal{K}}^{i}(\mathcal{F})\right), & & 0 \leqslant i \leqslant 2 \\
h^{j}(f, \mathcal{F}) & =\operatorname{dim} H_{c}^{j}\left(\mathbf{A}^{2} \times \overline{\mathbf{F}}_{q}, p_{2}^{*} \mathcal{F} \otimes \mathcal{L}_{\psi(f)}\right), & & 0 \leqslant j \leqslant 4 \\
m(f, \mathcal{F}) & =\operatorname{rank}\left(T_{\mathcal{K}}^{1}(\mathcal{F})\right)+\operatorname{pct}\left(T_{\mathcal{K}}^{1}(\mathcal{F})\right) . & &
\end{aligned}
$$

The proof of Theorems 2.3 and 2.5 will be based on the following steps:

Proposition 6.4. - The following assertions are true:

(1) The map $(f, \mathcal{F}) \mapsto c_{0}(f, \mathcal{F})$ is continuous.

(2) For $0 \leqslant j \leqslant 4$, the map $f \mapsto h^{j}\left(f, \overline{\mathbf{Q}}_{\ell}\right)=\operatorname{dim} H_{c}^{j}\left(\mathbf{A}^{2} \times \overline{\mathbf{F}}_{q}, \mathcal{L}_{\psi(f)}\right)$ is continuous.

(3) (a) If $f \mapsto h^{2}\left(f, \overline{\mathbf{Q}}_{\ell}\right)$ is continuous, then $f \mapsto c_{2}\left(f, \overline{\mathbf{Q}}_{\ell}\right)$ is continuous;

(b) if $(f, \mathcal{F}) \mapsto h^{2}(f, \mathcal{F})$ is continuous, then $(f, \mathcal{F}) \mapsto c_{2}(f, \mathcal{F})$ is continuous.

(4) If $f \mapsto c_{1}\left(f, \overline{\mathbf{Q}}_{\ell}\right)$ and $f \mapsto c_{2}\left(f, \overline{\mathbf{Q}}_{\ell}\right)$ are continuous, then $(f, \mathcal{F}) \mapsto$ $h^{2}(f, \mathcal{F})$ is continuous.

(5) (a) If $f \mapsto c_{2}\left(f, \overline{\mathbf{Q}}_{\ell}\right)$ is continuous, then $f \mapsto m\left(f, \overline{\mathbf{Q}}_{\ell}\right)$ is continuous;

(b) if $(f, \mathcal{F}) \mapsto c_{2}(f, \mathcal{F})$ is continuous, then $(f, \mathcal{F}) \mapsto m(f, \mathcal{F})$ is continuous.

(6) (a) If $f \mapsto m\left(f, \overline{\mathbf{Q}}_{\ell}\right)$ and $f \mapsto h^{2}\left(f, \overline{\mathbf{Q}}_{\ell}\right)$ are both continuous, then $f \mapsto c_{1}\left(f, \overline{\mathbf{Q}}_{\ell}\right)$ is continuous;

(b) if $(f, \mathcal{F}) \mapsto m(f, \mathcal{F})$ and $(f, \mathcal{F}) \mapsto h^{2}(f, \mathcal{F})$ are both continuous, then $(f, \mathcal{F}) \mapsto c_{1}(f, \mathcal{F})$ is continuous.

(7) If $(f, \mathcal{F}) \mapsto c_{i}(f, \mathcal{F})$ is continuous for $0 \leqslant i \leqslant 2$, then $(f, \mathcal{F}) \mapsto$ $h^{j}(f, \mathcal{F})$ is continuous for all $0 \leqslant j \leqslant 4$.

We now explain how to deduce Theorems 2.3 and 2.5 from this proposition. Since this may also look like spaghetti-mathematics, the reader may also wish to go straight to Sections 7 and 14 (possibly in the opposite order) which together give an account of the proof for the special case of the Fourier transform (and discuss another example arising in the POLYMATH8 project), in which case the flow of the proof is much easier to follow.

First of all, $c_{0}$ is continuous by (1), so we must show that $c_{1}, c_{2}$ and the $h^{j}$ are continuous.

Step 1. - Using (2), we can apply (3a) and deduce that $f \mapsto c_{2}\left(f, \overline{\mathbf{Q}}_{\ell}\right)$ is continuous. By $(5 \mathrm{a})$, it follows that $m\left(f, \overline{\mathbf{Q}}_{\ell}\right)$ is continuous. Combining this with (6a) and (2) again, we deduce that $f \mapsto c_{1}\left(f, \overline{\mathbf{Q}}_{\ell}\right)$ is continuous. 
At this point, we have proved both theorems in the special case when $\mathcal{F}=\overline{\mathbf{Q}}_{\ell}$ is the trivial sheaf.

Step 2. - From $(4)$ and Step 1 , we see that $(f, \mathcal{F}) \mapsto h^{2}(f, \mathcal{F})$ is continuous. This fact combined with (3b) shows that $(f, \mathcal{F}) \mapsto c_{2}(f, \mathcal{F})$ is continuous. In turn, $(5 \mathrm{~b})$ then proves that $(f, \mathcal{F}) \mapsto m(f, \mathcal{F})$ is continuous, and finally (6b) allows us to conclude that $(f, \mathcal{F}) \mapsto c_{1}(f, \mathcal{F})$ is continuous.

At this point we have proved Theorem 2.3 (and the continuity of $(f, \mathcal{F}) \mapsto$ $h^{2}(f, \mathcal{F})$ ); by $(7)$, we deduce that all $h^{j}$ are continuous.

\section{Remark 6.5.}

(1) We will in fact establish (3b) and (3a) directly by proving a direct relation between $c_{2}(f, \mathcal{F})$ and $h^{2}(f, \mathcal{F})$ for $\mathcal{F}=\overline{\mathbf{Q}}_{\ell}$ or in general, and similarly for (5b) and (5a), (6b) and (6a).

(2) The most crucial points in Proposition 6.4 are

- (2), which gives the starting point of the argument for the trivial sheaf, and which comes from the bounds for Betti numbers of Bombieri, Adolphson-Sperber and Katz.

- (4), which allows us to pass from properties known for the trivial sheaf only, to properties of all sheaves.

(3) It is only in the proof of (5b) and (5a) that we will use the restriction that continuity applies to $f$ with $\mathbf{c}(f)<p$.

\section{Spectral sequence argument}

We state here the few simple facts about spectral sequences that we require. We first recall the basic formalism, referring to [18, Appendix B] for a survey and [21, Chap. 10] for details.

Let $k$ be a fixed field. A converging (first quadrant) spectral sequence

$$
E_{2}^{p, q} \Longrightarrow E^{n},
$$

of $k$-vector spaces involves

(1) vector spaces $E_{2}^{p, q}$ defined for $p, q \geqslant 0$;

(2) vector spaces $E^{n}$ defined for $n \geqslant 0$;

(3) linear maps

$$
d_{2}^{p, q}: E_{2}^{p, q} \longrightarrow E_{2}^{p+2, q-1},
$$

(called differentials) ${ }^{(2)}$ for all $p$ and $q$ (with the convention $E_{2}^{p, q}=0$ if $p$ or $q$ is negative), such that

$$
d_{2}^{p, q} \circ d_{2}^{p-2, q+1}=0 .
$$

(2) Note that these differentials show that $p$ and $q$ do not play symmetric roles. 
Remark 7.1. - The use of the indices $p$ and $q$ for the spectral sequence is almost universal, although it clashes with the usual convention that $p$ is a prime and $q$ a power of $p$. We will use $i$ and $j$ instead of $p$ and $q$ when both notation are involved, although the difference in context should avoid confusion.

One defines

$$
E_{3}^{p, q}=\operatorname{ker} d_{2}^{p, q} / \operatorname{im} d_{2}^{p+2, q-1},
$$

and one shows that there are linear maps

$$
d_{3}^{p, q}: E_{3}^{p, q} \longrightarrow E_{3}^{p+3, q-2}
$$

such that $d_{3}^{p, q} \circ d_{3}^{p-3, q+2}=0$. This process is then suitably iterated to obtain $E_{j}^{p, q}$ for all $j \geqslant 2$, and differentials

$$
d_{j}^{p, q}: E_{j}^{p, q} \longrightarrow E_{j}^{p+j, q-j+1}
$$

(with composites vanishing).

One says that the spectral sequence degenerates at the $E_{j}$-level (where $j=2$ or 3 ) if $d_{i}^{p, q}=0$ for all $p, q \geqslant 0$ and $i \geqslant j$. When this is the case, the formalism gives (among other things) the following relation between the $E_{j}^{p, q}$ and the spaces $E^{n}$ : we have for all $n \geqslant 0$, a (non-canonical) isomorphism

$$
E^{n} \simeq \bigoplus_{p=0}^{n} E_{j}^{p, n-p},
$$

of $k$-vector spaces. (There is often more structure involved, but this will suffice for us.)

Furthermore, whether the spectral sequence degenerates at the $E_{2}$ or $E_{3}$ level or not, there is an exact sequence

$$
0 \longrightarrow E_{2}^{1,0} \longrightarrow E^{1} \longrightarrow E_{2}^{0,1} \longrightarrow E_{2}^{2,0}
$$

All these facts are stated in [18, p. 307-309]. The next proposition then summarizes all results we will need from spectral sequences:

Proposition 7.2. - Let $k$ be a field and let

$$
E_{2}^{p, q} \Longrightarrow E^{n}
$$

be a converging spectral sequence as above. Assume that $E_{2}^{p, q}=0$ unless $0 \leqslant p \leqslant 2$ and $0 \leqslant q \leqslant 2$.

(1) The spectral sequence degenerates at the $E_{3}$-level and we have

$$
E^{2} \simeq E_{3}^{0,2} \oplus E_{2}^{1,1} \oplus E_{3}^{2,0} .
$$


(2) We have

$$
\operatorname{dim} E^{n} \leqslant \sum_{p=0}^{n} \operatorname{dim} E_{2}^{p, n-p}
$$

and

$$
\operatorname{dim} E_{2}^{0,2} \leqslant \operatorname{dim} E^{2}+\operatorname{dim} E_{2}^{2,1} .
$$

(3) Assume in addition that $E_{2}^{p, q}=0$ if $q=0$. We have then $E_{2}^{0,1} \simeq E^{1}$.

\section{Proof.}

(1). - From (7.1), we see that $E_{3}^{p, q}=0$ unless $0 \leqslant p, q \leqslant 2$ since it is a quotient of a subspace of $E_{2}^{p, q}$. But then (7.2) shows that, for any $p, q$, either the source of the target of $d_{3}^{p, q}$ is zero. In fact, for all $i \geqslant 3$, either the target or the source of $d_{i}^{p, q}$ vanishes, and therefore the spectral sequence degenerates at that level.

By (7.3) we deduce that

$$
E^{2} \simeq E_{3}^{0,2} \oplus E_{3}^{1,1} \oplus E_{3}^{0,2},
$$

but

$$
E_{3}^{1,1}=\operatorname{ker} d_{2}^{1,1} / \operatorname{im} d_{2}^{-1,2},
$$

and since $d_{2}^{1,1}$ and $d_{2}^{-1,2}$ are both zero (the target of the first and the source of the second are zero), we have $E_{3}^{1,1}=E_{2}^{1,1}$, hence (7.5).

(2). - By (1) and (7.3), we have

$$
E^{n} \simeq E_{3}^{n, 0} \oplus E_{3}^{n-1,1} \oplus \cdots \oplus E_{3}^{0, n} .
$$

Since

$$
\operatorname{dim} E_{3}^{p, q} \leqslant \operatorname{dim} E_{2}^{p, q},
$$

for all $p$ and $q$, by (7.1), we obtain

$$
\operatorname{dim} E^{n}=\sum_{p=0}^{n} \operatorname{dim} E_{3}^{p, q} \leqslant \sum_{p=0}^{n} \operatorname{dim} E_{2}^{p, q} .
$$

Similarly, we note that

$$
E_{3}^{0,2}=\operatorname{ker} d_{2}^{0,2} / \operatorname{im} d_{2}^{-2,1}=\operatorname{ker} d_{2}^{0,2},
$$

and hence we have a short exact sequence

$$
0 \longrightarrow E_{3}^{0,2} \longrightarrow E_{2}^{0,2} \stackrel{d_{2}^{0,2}}{\longrightarrow} E_{2}^{2,1}
$$

which implies that

$$
\operatorname{dim} E_{2}^{0,2} \leqslant \operatorname{dim} E_{3}^{0,2}+\operatorname{dim} E_{2}^{2,1} .
$$


From the degeneracy at the $E_{3}$-level, we then get

$$
\operatorname{dim} E_{3}^{0,2} \leqslant \operatorname{dim} E^{2}
$$

hence the bound for $E_{2}^{0,2}$.

(3). - The exact sequence (7.4), under the assumptions that $E_{2}^{p, 0}=0$, becomes

$$
0 \longrightarrow E^{1} \longrightarrow E_{2}^{0,1} \longrightarrow 0,
$$

hence the result.

The spectral sequences we use are given by the following lemma:

Lemma 7.3. - Let $\mathbf{F}_{q}$ be a finite field of characteristic $p, \ell \neq p$ a prime number. Let $f \in \mathbf{F}_{q}(X, Y)$ be a rational function, and denote

$$
\mathcal{K}=\mathcal{L}_{\psi(f(X, Y))}
$$

where $\psi$ is a non-trivial additive $\ell$-adic character. Denote $f^{*}(X, Y)=$ $f(Y, X) \in \mathbf{F}_{q}(X, Y)$, and

$$
\mathcal{K}^{*}=\mathcal{L}_{\psi\left(f^{*}\right)} .
$$

Let $\mathcal{F}$ be a constructible $\ell$-adic sheaf on $\mathbf{A}_{\mathbf{F}_{q}}^{1}$.

(1) For any dense open subsets $U, V$ of $\mathbf{A}_{\mathbf{F}_{q}}^{1}$, with $p_{1}, p_{2}$ denoting the projection maps $U \times V \rightarrow U$ and $U \times V \rightarrow V$, respectively, there exist converging spectral sequences

$$
\begin{aligned}
& E_{2}^{i, j}=H_{c}^{i}\left(\bar{U}, T_{\mathcal{K}}^{j}(\mathcal{F})\right) \Longrightarrow H_{c}^{i+j}\left(\bar{U} \times \mathbf{A}^{1}, p_{2}^{*} \mathcal{F} \otimes \mathcal{K}\right), \\
& E_{2}^{i, j}=H_{c}^{i}\left(\bar{V}, \mathcal{F} \otimes T_{\mathcal{K}^{*}}^{j}\left(\overline{\mathbf{Q}}_{\ell}\right)\right) \Longrightarrow H_{c}^{i+j}\left(\mathbf{A}^{1} \times \bar{V}, p_{2}^{*} \mathcal{F} \otimes \mathcal{K}\right) \\
& \text { of } \overline{\mathbf{Q}}_{\ell^{-} \text {-vector spaces }}
\end{aligned}
$$

(2) These two spectral sequences satisfy $E_{2}^{i, j}=0$ unless $0 \leqslant i \leqslant 2$ and $1 \leqslant j \leqslant 2$.

\section{Proof.}

(1). - The first spectral sequence is the Leray spectral sequence of the first projection map $p_{1}: U \times \mathbf{A}^{1} \rightarrow U$ and of the sheaf $p_{2}^{*} \mathcal{F} \otimes \mathcal{K}$ (see, e.g., [7, Thm. 7.4.4(ii)] or [18, Thm. VI.3.2(c)]).

The second spectral sequence arises from the Leray spectral sequence of the second projection $p_{2}: \mathbf{A}^{1} \times V \rightarrow V$ and of the sheaf $p_{2}^{*} \mathcal{F} \otimes \mathcal{K}$, namely

$$
E_{2}^{i, j}=H_{c}^{i}\left(\bar{V}, R^{j} p_{2, !}\left(p_{2}^{*} \mathcal{F} \otimes \mathcal{K}\right)\right) \Longrightarrow H_{c}^{i+j}\left(\mathbf{A}^{1} \times \bar{V}, p_{2}^{*} \mathcal{F} \otimes \mathcal{K}\right)
$$

together with the facts that

$$
R^{j} p_{2, !}\left(p_{2}^{*} \mathcal{F} \otimes \mathcal{K}\right)=\mathcal{F} \otimes R^{j} p_{2, !}\left(\mathcal{L}_{\psi(f(X, Y))}\right)
$$

by the projection formula (see, e.g., [7, Thm. 7.4.7]), and that we can identify $R^{j} p_{2, !}\left(\mathcal{L}_{\psi(f(X, Y))}\right)$ with $T_{\mathcal{K}^{*}}^{j}\left(\overline{\mathbf{Q}}_{\ell}\right)$ (restricted to $\left.V\right)$. 
(2). - The fact that $E_{2}^{i, j}=0$ unless $0 \leqslant i, j \leqslant 2$ is immediate from (1) and from the vanishing of cohomology of curves (resp. of higher-direct image sheaves for maps with curves as fibers) in Proposition 4.1(1): the former constrains $i$ to be between 0 and 2, and the second constrains similarly $j$.

For the vanishing when $j=0$, we note that the stalk at $x \in \mathbf{A}^{1}\left(\overline{\mathbf{F}}_{q}\right)$ of $R^{0} p_{1, !}\left(p_{2}^{*} \mathcal{F} \otimes \mathcal{K}\right)$ is, by the proper base change theorem, equal to

$$
H_{c}^{0}\left(\mathbf{A}^{1} \times \overline{\mathbf{F}}_{q}, \mathcal{F} \otimes \mathcal{L}_{\psi(f(x, Y))}\right)=0
$$

by Lemma 4.2. Similarly, the stalk of $R^{0} p_{2, !}(\mathcal{K})$ at $y$ is

$$
H_{c}^{0}\left(\mathbf{A}^{1} \times \overline{\mathbf{F}}_{q}, \mathcal{L}_{\psi(f(X, y))}\right)=0,
$$

and these facts show that $E_{2}^{i, 0}=0$ for all $i$ in both spectral sequences.

\section{Beginning of the proof}

We will now begin the proof of Proposition 6.4. As a warm-up, the reader may wish to have a look at Section 14 where we discuss the simpler case of the Fourier transform (where $f(X, Y)=X Y$ ) and a closely related case appearing in the POLYMATH8 project.

We first deal with parts (1) and (2) of Proposition 6.4.

(1). - We claim that $T_{\mathcal{K}}^{0}(\mathcal{F})=0$ for all $f$ and $\mathcal{F}$. Indeed, by the proper base change theorem (Proposition 4.1(4)), the stalk of $R^{0} p_{1, !}\left(p_{2}^{*} \mathcal{F} \otimes \mathcal{K}\right)$ over $x \in \mathbf{A}^{1}\left(\overline{\mathbf{F}}_{q}\right)$ is

$$
H_{c}^{0}\left(\mathbf{A}^{1} \times \overline{\mathbf{F}}_{q}, \mathcal{F} \otimes \mathcal{L}_{\psi(f(x, Y))}\right)=0
$$

by Lemma 4.2 .

(2). - By the bounds of Bombieri, Adolphson-Sperber and Katz (see, e.g., [14, Thm. 12]), the sum of Betti numbers

$$
\sum_{i=0}^{4} \operatorname{dim} H_{c}^{i}\left(\mathbf{A}^{2} \times \overline{\mathbf{F}}_{q}, \mathcal{L}_{\psi(f)}\right)
$$

is bounded by $(1+\mathbf{c}(f))^{B}$ for some absolute constant $B \geqslant 1$, which proves the continuity of $h^{i}\left(f, \overline{\mathbf{Q}}_{\ell}\right)$. Precisely, in order to apply the result of Katz, one writes $f=f_{1} / f_{2}$ with $f_{i} \in \mathbf{F}_{q}[X, Y]$ and $f_{1}$ coprime to $f_{2}$, then one notes that if $U_{2} \subset \mathbf{A}^{2}$ is the open subset where the denominator $f_{2}$ is invertible, we have

$$
H_{c}^{i}\left(\mathbf{A}^{2} \times \overline{\mathbf{F}}_{q}, \mathcal{L}_{\psi(f)}\right)=H_{c}^{i}\left(U_{2} \times \overline{\mathbf{F}}_{q}, \mathcal{L}_{\psi(f)}\right)
$$


by definition of cohomology with compact support. Define $Z \subset \mathbf{A}^{3}$, where $\mathbf{A}^{3}$ has coordinates $(U, X, Y)$, to be the zero set of the polynomial $U f_{2}(X, Y)-1$. Then the morphism

$$
\alpha\left\{\begin{array}{l}
Z \rightarrow U_{2} \\
(u, x, y) \mapsto(x, y)
\end{array}\right.
$$

is an isomorphism such that $\alpha^{*} \mathcal{L}_{\psi(f)}$ is isomorphic to the lisse sheaf $\mathcal{L}_{\psi}(\widetilde{f})$ for the polynomial $\tilde{f}=U f_{1}(X, Y) \in \mathbf{F}_{q}[U, X, Y]$. Katz's theorem gives precisely the upper-bound

$$
\sum_{i=0}^{4} \operatorname{dim} H_{c}^{i}\left(\bar{Z}, \mathcal{L}_{\psi(\tilde{f})}\right) \leqslant 3\left(1+1+\max \left(1+\operatorname{deg} f_{1}, 1+\operatorname{deg} f_{2}\right)\right)^{3+1},
$$

and hence the result.

The other parts of the proof are more involved, and require the tools of Section 7. However, before going further we will deal directly with the special case when $f \in \mathbf{F}_{q}(X)+\mathbf{F}_{q}(Y)$ (the reader is invited to figure out the analogue of Section 3 in this case).

\subsection{Proof of Theorems 2.3 and 2.5 in a factorable case}

So assume that

$$
f=f_{1}+f_{2},
$$

with $f_{1} \in \mathbf{F}_{q}(X)$ and $f_{2} \in \mathbf{F}_{q}(Y)$. We have $\mathcal{K}=p_{1}^{*} \mathcal{L}_{1} \otimes p_{2}^{*} \mathcal{L}_{2}$, where $\mathcal{L}_{i}=$ $\mathcal{L}_{\psi\left(f_{i}\right)}$, hence

$$
R^{i} p_{1, !}\left(p_{2}^{*} \mathcal{F} \otimes p_{2}^{*} \mathcal{L}_{2} \otimes p_{1}^{*} \mathcal{L}_{1}\right) \simeq \mathcal{L}_{1} \otimes R^{i} p_{1, !}\left(p_{2}^{*}\left(\mathcal{F} \otimes \mathcal{L}_{2}\right)\right),
$$

for $0 \leqslant i \leqslant 2$, by the projection formula (see, e.g., [7, Thm. 7.4.7]).

But the sheaf $R^{i} p_{1, !}\left(p_{2}^{*}\left(\mathcal{F} \otimes \mathcal{L}_{2}\right)\right)$ is the constant sheaf associated to $H_{c}^{i}\left(\mathbf{A}^{1} \times \overline{\mathbf{F}}_{q}, \mathcal{F} \otimes \mathcal{L}_{2}\right)$ : indeed, applying [2, Arcata, IV, Thm. 5.4] to the cartesian diagram

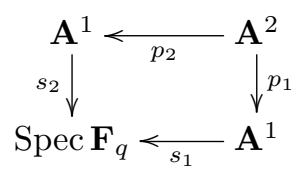

and the sheaf $\mathcal{F} \otimes \mathcal{L}_{2}$ on $\mathbf{A}^{1}$, we obtain

$$
s_{1}^{*} R^{i} s_{2, !}\left(\mathcal{F} \otimes \mathcal{L}_{2}\right) \simeq R^{i} p_{1, !}\left(p_{2}^{*}\left(\mathcal{F} \otimes \mathcal{L}_{2}\right)\right),
$$

and the left-hand side is a constant sheaf (since it is pulled-back from $\mathbf{F}_{q}$ ) and has fiber $R^{i} s_{2, !}\left(\mathcal{F} \otimes \mathcal{L}_{2}\right)=H_{c}^{i}\left(\mathbf{A}^{1} \times \overline{\mathbf{F}}_{q}, \mathcal{F} \otimes \mathcal{L}_{2}\right)$, by the definition of cohomology with compact support and higher-direct images. 
Hence we have (see (2.1))

$$
c_{i}\left(f_{1}+f_{2}, \mathcal{F}\right) \leqslant\left(\operatorname{dim} H_{c}^{i}\left(\mathbf{A}^{1} \times \overline{\mathbf{F}}_{q}, \mathcal{F} \otimes \mathcal{L}_{2}\right)\right) \times \mathbf{c}\left(\mathcal{L}_{1}\right),
$$

which is continuous as a function of $\mathbf{c}(\mathcal{F})$ and $\mathbf{c}(f)$ by Lemmas 4.3 and 4.4.

This proves Theorem 2.3 in the special case $f \in \mathbf{F}_{q}(X)+\mathbf{F}_{q}(Y)$, and Theorem 2.5 follows either from the argument in Section 13 (which is general) or from an application of the Künneth formula (see, e.g., [2, Sommes Trig., $\left.(2.4)^{*}\right]$ ) and of Lemma 4.3.

Remark 8.1. - In particular, by the definition of the conductor, we have established Proposition 6.4 in the case $f \in \mathbf{F}_{q}(X)+\mathbf{F}_{q}(Y)$. In the sequel, we may (and will) assume that

$$
f \notin \mathbf{F}_{q}(X)+\mathbf{F}_{q}(Y) .
$$

\section{Proposition 6.4: proof of (3b) and (3a)}

We prove $(3 \mathrm{~b})$ and assume that the function $(f, \mathcal{F}) \mapsto h^{2}(f, \mathcal{F})$ is continuous, and our aim is to show that

$$
(f, \mathcal{F}) \mapsto c_{2}(f, \mathcal{F})=\mathbf{c}\left(T_{\mathcal{K}}^{2}(\mathcal{F})\right)
$$

is continuous. As pointed out in Remark 8.1, we can assume from now on that

$$
f \notin \mathbf{F}_{q}(X)+\mathbf{F}_{q}(Y) .
$$

Lemma 9.1. - Assume $f \notin \mathbf{F}_{q}(X)+\mathbf{F}_{q}(Y)$. Then $T_{\mathcal{K}}^{2}(\mathcal{F})$ vanishes generically.

Proof. - Denote

$$
\mathcal{G}=T_{\mathcal{K}}^{2}(\mathcal{F})=R^{2} p_{1, !}\left(p_{2}^{*} \mathcal{F} \otimes \mathcal{K}\right) .
$$

Let $\left(\mathcal{F}_{i}\right)$ be the (geometric) Jordan-Hölder factors of $\mathcal{F}$; then the geometric Jordan-Hölder factors of $p_{2}^{*} \mathcal{F} \otimes \mathcal{K}$ are the $p_{2}^{*} \mathcal{F}_{i} \otimes \mathcal{K}$, so that we may assume that $\mathcal{F}$ is geometrically irreducible.

Let $\eta=\operatorname{Spec}\left(\mathbf{F}_{q}(X)\right)$ be the generic point of the affine line $\mathbf{A}_{\mathbf{F}_{q}}^{1}$ (with coordinate $X)$, let $\bar{\eta}=\operatorname{Spec}\left(\overline{\mathbf{F}_{q}(X)}\right)$ be a geometric point above $\eta$. By constructibility, the stalks of $\mathcal{G}$ vanish for all $x$ in a dense open subset if and only if the stalk $\mathcal{G}_{\bar{\eta}}$ is zero.

By the proper base change theorem, we have

$$
\mathcal{G}_{\bar{\eta}}=H_{c}^{2}\left(\mathbf{A}^{1} \times \overline{\mathbf{F}_{q}(X)}, \mathcal{F} \otimes \mathcal{L}_{\psi\left(f_{X}(Y)\right)}\right),
$$


where $f_{X}(Y)=f(X, Y)$. Assume this stalk is non-zero. Then, using the coinvariant formula for the second cohomology group on a curve, it follows that there exists an open subset $U$ of the affine line (with coordinate $Y$ ) over $\overline{\mathbf{F}_{q}(X)}$ such that

$$
\mathcal{F} \simeq \mathcal{L}_{\psi\left(-f_{X}(Y)\right)}
$$

as sheaves on $U \times \overline{\mathbf{F}_{q}(X)}$. Since they are middle-extension sheaves, they are isomorphic as sheaves on the affine line over $\overline{\mathbf{F}_{q}(X)}$.

Note that $\mathcal{F}$ is pulled back from the affine line $\mathbf{A}^{1}$ over $\mathbf{F}_{q}$ (still with coordinate $Y$ ), and so the classification of Artin-Schreier sheaves shows that $f$ is, up to an additive "constant" in $\overline{\mathbf{F}_{q}(X)}$, an element in $\mathbf{F}_{q}(Y)$, i.e., we have

$$
f=f_{1}+f_{2},
$$

with $f_{1} \in \mathbf{F}_{q}(X)$ and $f_{2} \in \mathbf{F}_{q}(Y)$.

Remark 9.2. - One can also prove this lemma using more elementary arguments on rational functions, by looking at the vanishing at individual stalks and the classification of Artin-Schreier sheaves on $\mathbf{A}_{\mathbf{F}_{q}}^{1}$.

Because of this lemma, the conductor of $\mathcal{G}=T_{\mathcal{K}}^{2}(\mathcal{F})$ is equal to $\operatorname{pct}(\mathcal{G})$ (the generic rank is 0 , and thus the action of all inertia groups on the generic fiber is trivial, which implies that $n(\mathcal{G})=0$ and hence the Swan conductors also vanish). Hence

$$
\mathbf{c}(\mathcal{G})=\operatorname{dim} H_{c}^{0}\left(\mathbf{A}^{1} \times \overline{\mathbf{F}}_{q}, T_{\mathcal{K}}^{2}(\mathcal{F})\right) .
$$

In the first spectral sequence of Lemma 7.3 , with $U=\mathbf{A}^{1}$, we must therefore bound $\operatorname{dim} E_{2}^{0,2}$. By the last part of Proposition 7.2(2), we have

$$
\begin{aligned}
\operatorname{dim} E_{2}^{0,2} & \leqslant \operatorname{dim} E^{2}+\operatorname{dim} E_{2}^{2,1} \\
& =\operatorname{dim} H_{c}^{2}\left(\mathbf{A}^{2} \times \overline{\mathbf{F}}_{q}, p_{2}^{*} \mathcal{F} \otimes \mathcal{K}\right)+\operatorname{dim} H_{c}^{2}\left(\mathbf{A}^{1} \times \overline{\mathbf{F}}_{q}, T_{\mathcal{K}}^{1}(\mathcal{F})\right) \\
& =h^{2}(f, \mathcal{F})+\operatorname{dim} H_{c}^{2}\left(\mathbf{A}^{1} \times \overline{\mathbf{F}}_{q}, T_{\mathcal{K}}^{1}(\mathcal{F})\right) .
\end{aligned}
$$

We have already recalled in the proof of Lemma 4.3 that

$$
\operatorname{dim} H_{c}^{2}\left(\mathbf{A}^{1} \times \overline{\mathbf{F}}_{q}, T_{\mathcal{K}}^{1}(\mathcal{F})\right) \leqslant \operatorname{rank}\left(T_{\mathcal{K}}^{1}(\mathcal{F})\right) .
$$

Using the notation of Definition 4.7 and the proper base change theorem, we get

$$
\begin{aligned}
\operatorname{dim} H_{c}^{2}\left(\mathbf{A}^{1} \times \overline{\mathbf{F}}_{q}, T_{\mathcal{K}}^{1}(\mathcal{F})\right) & \leqslant \max _{x \in \mathbf{A}^{1}\left(\overline{\mathbf{F}}_{q}\right)} \operatorname{dim} T_{\mathcal{K}}^{1}(\mathcal{F})_{x} \\
& \leqslant \max _{x \in \overline{\mathbf{F}}_{q}} \operatorname{dim} H_{c}^{1}\left(\mathbf{A}^{1} \times \overline{\mathbf{F}}_{q}, \mathcal{F} \otimes \mathcal{L}_{x}\right),
\end{aligned}
$$


and by Corollary 4.10 , this shows that $(f, \mathcal{F}) \mapsto \operatorname{dim} H_{c}^{2}\left(\mathbf{A}^{1} \times \overline{\mathbf{F}}_{q}, T_{\mathcal{K}}^{1}(\mathcal{F})\right)$ is continuous. The inequality (9.1) then finishes the proof of $(3 \mathrm{~b})$.

The proof of (3a) is identical: it suffices to fix $\mathcal{F}=\overline{\mathbf{Q}}_{\ell}$ in the above argument.

\section{Proposition 6.4: proof of (4)}

We assume that the function $f \mapsto c_{i}\left(f, \overline{\mathbf{Q}}_{\ell}\right)$ are continuous for $i=1$ and $i=2$, and aim at proving that $(f, \mathcal{F}) \mapsto h^{2}(f, \mathcal{F})$ is continuous.

We apply the second spectral sequence of Lemma 7.3, with $V=\mathbf{A}^{1}$, and the first part of Proposition 7.2(2) with $n=2$ : this gives

$$
h^{2}(f, \mathcal{F})=\operatorname{dim} E^{2} \leqslant \operatorname{dim} E_{2}^{2,0}+\operatorname{dim} E_{2}^{1,1}+\operatorname{dim} E_{2}^{0,2},
$$

where

$$
E_{2}^{i, j}=H_{c}^{i}\left(\mathbf{A}^{1} \times \overline{\mathbf{F}}_{q}, \mathcal{F} \otimes T_{\mathcal{K}^{*}}^{j}\left(\overline{\mathbf{Q}}_{\ell}\right)\right)
$$

We note that $\mathbf{c}\left(\mathcal{K}^{*}\right)=\mathbf{c}(\mathcal{K})$. We have $E_{2}^{2,0}=0$ (cf. (1) of Section 8), and

$$
\operatorname{dim} E_{2}^{1,1}=\operatorname{dim} H_{c}^{1}\left(\mathbf{A}^{1} \times \overline{\mathbf{F}}_{q}, \mathcal{F} \otimes T_{\mathcal{K}^{*}}^{1}\left(\overline{\mathbf{Q}}_{\ell}\right)\right)
$$

is continuous by Lemmas 4.3 and 4.4 , since the conductor of $T_{\mathcal{K}^{*}}^{1}\left(\overline{\mathbf{Q}}_{\ell}\right)$ is bounded polynomially in terms of the conductor of $f$ by assumption.

Finally, we have

$$
\operatorname{dim} E_{2}^{0,2}=\operatorname{dim} H_{c}^{0}\left(\mathbf{A}^{1} \times \overline{\mathbf{F}}_{q}, \mathcal{F} \otimes T_{\mathcal{K}^{*}}^{2}\left(\overline{\mathbf{Q}}_{\ell}\right)\right) \leqslant \mathbf{c}\left(\mathcal{F} \otimes T_{\mathcal{K}^{*}}^{2}\left(\overline{\mathbf{Q}}_{\ell}\right)\right) .
$$

By assumption, $f \mapsto c_{2}\left(f^{*}, \overline{\mathbf{Q}}_{\ell}\right)$ is continuous, and therefore the function $\operatorname{dim} E_{2}^{0,2}$ is continuous (Lemma 4.4). Thus $h^{2}(f, \mathcal{F})$ is also continuous.

\section{Proposition 6.4: proof of (5b) and (5a)}

We assume that the function $(f, \mathcal{F}) \mapsto c_{2}(f, \mathcal{F})$ is continuous, and aim at proving that $(f, \mathcal{F}) \mapsto m(f, \mathcal{F})$ is continuous. We still assume that $f \notin$ $\mathbf{F}_{q}(X)+\mathbf{F}_{q}(Y)$.

We set

$$
\mathcal{G}=T_{\mathcal{K}}^{1}(\mathcal{F})=R^{1} p_{1, !}\left(p_{2}^{*} \mathcal{F} \otimes \mathcal{K}\right) .
$$

We have to bound the $\operatorname{rank} \operatorname{rank}(\mathcal{G})$ and the punctual part $\operatorname{pct}(\mathcal{G})$. 


\subsection{Bounding $\operatorname{rank}(\mathcal{G})$}

For $x \in \mathbf{A}^{1}\left(\overline{\mathbf{F}}_{q}\right)$, the stalk of $\mathcal{G}$ at $x$ is

$$
\mathcal{G}_{x}=H_{c}^{1}\left(\mathbf{A}^{1} \times \overline{\mathbf{F}}_{q}, \mathcal{F} \otimes \mathcal{L}_{x}\right)
$$

by the proper base change theorem. The generic rank of $\mathcal{G}$ is at most the maximal value of the dimension of this stalk as $x$ varies. Hence, by Corollary 4.10 , it is a continuous function of $(f, \mathcal{F})$.

\subsection{Bounding $\operatorname{pct}(\mathcal{G})$}

We have

$$
\operatorname{pct}(\mathcal{G})=\operatorname{dim} H_{c}^{0}\left(\mathbf{A}^{1} \times \overline{\mathbf{F}}_{q}, \mathcal{G}\right) .
$$

Again by (1) of Section 8, we have $T_{\mathcal{K}}^{0}(\mathcal{F})=0$, therefore in the first spectral sequence of Lemma 7.3 (with $U=\mathbf{A}^{1}$ ) we have $E_{2}^{p, 0}=0$ so that applying Proposition $7.2(3)$, we obtain

$$
\operatorname{pct}(\mathcal{G})=\operatorname{dim} H_{c}^{1}\left(\mathbf{A}^{2} \times \overline{\mathbf{F}}_{q}, p_{2}^{*} \mathcal{F} \otimes \mathcal{K}\right) .
$$

To bound this last quantity, we need the following cohomological lemma:

LEMMA 11.1. - Let $\mathbf{F}_{q}$ be a finite field of characteristic $p, \ell \neq p a$ prime number and $\psi$ a non-trivial $\ell$-adic additive character. Let $f=g_{1} / g_{2} \in$ $\mathbf{F}_{q}(X, Y)$ be a rational function with $g_{1}, g_{2} \in \mathbf{F}_{q}[X, Y]$ coprime and

$$
\mathcal{K}=\mathcal{L}_{\psi(f)} .
$$

Let $\mathcal{F}$ be a constructible $\ell$-adic sheaf on $\mathbf{A}_{\mathbf{F}_{q}}^{1}$.

Let $C$ be the union of the zero set of $g_{2}$, seen as a reduced subscheme of $\mathbf{A}^{2}$, and of the lines

$$
\mathbf{A}^{1} \times\{y\} \subset \mathbf{A}^{2},
$$

where $y \in \mathbf{A}^{1}\left(\overline{\mathbf{F}}_{q}\right)$ is a singularity of $\mathcal{F}$. Let $W \subset \mathbf{A}^{2}$ be the open subset complement of $C$.

(1) We have

$$
H_{c}^{1}\left(W \times \overline{\mathbf{F}}_{q}, p_{2}^{*} \mathcal{F} \otimes \mathcal{K}\right)=0
$$

(2) The map

$$
(f, \mathcal{F}) \longmapsto \operatorname{dim} H_{c}^{1}\left(C \times \overline{\mathbf{F}}_{q}, p_{2}^{*} \mathcal{F} \otimes \mathcal{K}\right)
$$

is continuous. 
We give the proof of this lemma below, but let us explain first how to conclude the proof of $(5 \mathrm{~b})$.

Let $W \subset \mathbf{A}^{2} \times \mathbf{F}_{q}$ be the (dense) open set defined in Lemma 11.1 and $C=\mathbf{A}^{2}-W$ its complement. By (1) of this Lemma, we have

$$
H_{c}^{1}\left(W \times \overline{\mathbf{F}}_{q}, p_{2}^{*} \mathcal{F} \otimes \mathcal{K}\right)=0
$$

and from the excision inequality (4.2), we get

$$
\operatorname{pct}(\mathcal{G})=\operatorname{dim} H_{c}^{1}\left(\mathbf{A}^{2} \times \overline{\mathbf{F}}_{q}, p_{2}^{*} \mathcal{F} \otimes \mathcal{K}\right) \leqslant \operatorname{dim} H_{c}^{1}\left(C \times \overline{\mathbf{F}}_{q}, p_{2}^{*} \mathcal{F} \otimes \mathcal{K}\right),
$$

and the second part of Lemma 11.1 shows that $(f, \mathcal{F}) \mapsto \operatorname{pct}(\mathcal{G})$ is continuous.

The proof of (5a) is identical: it suffices to fix $\mathcal{F}=\overline{\mathbf{Q}}_{\ell}$ in the above argument.

Proof of Lemma 11.1.

(1). - The open subset $W$ is a smooth affine surface, and $p_{2}^{*} \mathcal{F} \otimes \mathcal{K}$ is lisse on $W$, so (4.4) gives the vanishing of the first cohomology group.

(2). - Write $C_{1}$ for the zero set of $g_{2}$ (as a reduced scheme) and

$$
C_{2}=\bigcup_{y \in \tilde{S}} \mathbf{A}^{1} \times\{y\}
$$

where $y$ ranges over those singularities of $\mathcal{F}$ in $\mathbf{A}^{1}\left(\overline{\mathbf{F}}_{q}\right)$ such that $\mathbf{A}^{1} \times\{y\}$ is not contained in $C_{1}$.

Let $S=C_{1} \cap C_{2}$ be the intersection of these two sets; because of the last restriction, this is a finite set, and its order is bounded polynomially in terms of $\mathbf{c}(\mathcal{F})$ and $\mathbf{c}(f)$ (e.g., by Bézout's Theorem for plane curves). Applying the excision exact sequence (4.1) to $C$ and the complement $U$ (in $C$ ) of the closed set $C_{1}$, we get by (4.2) the bound

$$
\begin{array}{r}
\operatorname{dim} H_{c}^{1}\left(\bar{C}, p_{2}^{*} \mathcal{F} \otimes \mathcal{K}\right) \leqslant \operatorname{dim} H_{c}^{1}\left(\bar{U}, p_{2}^{*} \mathcal{F} \otimes \mathcal{K}\right)+\operatorname{dim} H_{c}^{1}\left(\bar{C}_{1}, p_{2}^{*} \mathcal{F} \otimes \mathcal{K}\right) \\
=\operatorname{dim} H_{c}^{1}\left(\bar{U}, p_{2}^{*} \mathcal{F} \otimes \mathcal{K}\right)
\end{array}
$$

since $\mathcal{K}$, by definition, is zero on $C_{1}$.

We have $U=C_{2}-S$, and we apply again the excision exact sequence to $C_{2}$ and its open set $U$, obtaining by (4.3) the bound

$$
\operatorname{dim} H_{c}^{1}\left(\bar{C}, p_{2}^{*} \mathcal{F} \otimes \mathcal{K}\right) \leqslant \operatorname{dim} H_{c}^{1}\left(\bar{U}, p_{2}^{*} \mathcal{F} \otimes \mathcal{K}\right) \leqslant \operatorname{dim} H_{c}^{1}\left(\bar{C}_{2}, p_{2}^{*} \mathcal{F} \otimes \mathcal{K}\right)
$$

(because $H_{c}^{0}\left(\bar{S}, p_{2}^{*} \mathcal{F} \otimes \mathcal{K}\right)=0$ since $S \subset C_{1}$, so that $\mathcal{K}$ is zero on $S$ ). Finally, we have

$$
H_{c}^{1}\left(\bar{C}_{2}, p_{2}^{*} \mathcal{F} \otimes \mathcal{K}\right)=\bigoplus_{y \in \tilde{S}} \mathcal{F}_{y} \otimes H_{c}^{1}\left(\mathbf{A}^{1} \times \overline{\mathbf{F}}_{q}, \mathcal{L}_{\psi(f(x, Y))}\right)
$$


and moreover both $\widetilde{S}$ and $S$ have order bounded in terms of $\mathbf{c}(\mathcal{F})$ and $\mathbf{c}(f)$, so that we obtain the result.

\section{Proposition 6.4: proof of (6b) and (6a)}

We assume that the functions $(f, \mathcal{F}) \mapsto m(f, \mathcal{F})$ and $(f, \mathcal{F}) \mapsto h^{2}(f, \mathcal{F})$ are continuous, and aim at proving that

$$
(f, \mathcal{F}) \longmapsto c_{1}(f, \mathcal{F})
$$

is continuous. We recall that

$$
\mathcal{G}=T_{\mathcal{K}}^{1}(\mathcal{F})=R^{1} p_{1, !}\left(p_{2}^{*} \mathcal{F} \otimes \mathcal{K}\right) .
$$

We have by definition

$$
\mathbf{c}(\mathcal{G})=\mathbf{c}\left(\mathcal{G}^{\prime}\right)+\operatorname{pct}(\mathcal{G})
$$

where $\mathcal{G}^{\prime}$ is the quotient of $\mathcal{G}$ by punctual sections. From the assumption that $m(f, \mathcal{F})$ is continuous, the punctual part $\operatorname{pct}(\mathcal{G})$ and $\operatorname{rank}\left(\mathcal{G}^{\prime}\right)$ are continuous; by Lemma 4.11 (applied to $\mathcal{G}^{\prime}$ ), it is enough to prove that

$$
(f, \mathcal{F}) \longmapsto \operatorname{dim} H_{c}^{1}\left(\mathbf{A}^{1} \times \overline{\mathbf{F}}_{q}, T_{\mathcal{K}}^{1}(\mathcal{F})\right)
$$

is continuous.

We use the first spectral sequence of Lemma 7.3 (with the open set $\mathbf{A}^{1}$ ). By Proposition 7.2(1) and (7.3), we have

$$
E^{2} \simeq E_{3}^{0,2} \oplus E_{2}^{1,1} \oplus E_{3}^{2,0}
$$

and in particular

$\operatorname{dim} H_{c}^{1}\left(\mathbf{A}^{1} \times \overline{\mathbf{F}}_{q}, T_{\mathcal{K}}^{1}(\mathcal{F})\right)=\operatorname{dim} E_{2}^{1,1} \leqslant \operatorname{dim} E^{2}=\operatorname{dim} H_{c}^{2}\left(\mathbf{A}^{2} \times \overline{\mathbf{F}}_{q}, p_{2}^{*} \mathcal{F} \otimes \mathcal{K}\right)$. Since $h^{2}(f, \mathcal{F})$ is also assumed to be continuous, this proves $(6 \mathrm{~b})$.

The proof of (6a) is identical: it suffices to fix $\mathcal{F}=\overline{\mathbf{Q}}_{\ell}$ in the above argument.

\section{Proposition 6.4: proof of (7)}

We assume that the functions $(f, \mathcal{F}) \mapsto c_{i}(f, \mathcal{F})$ are continuous for $0 \leqslant$ $i \leqslant 2$, and aim at proving that

$$
(f, \mathcal{F}) \longmapsto h^{j}(f, \mathcal{F})
$$

is continuous for $j \leqslant 4$. 
We use the first spectral sequence of Lemma 7.3 with $U=\mathbf{A}^{1}$. For any $j$, it implies that

$$
h^{j}(f, \mathcal{F})=\operatorname{dim} E^{j} \leqslant \sum_{p=0}^{j} \operatorname{dim} H_{c}^{p}\left(\mathbf{A}^{1} \times \overline{\mathbf{F}}_{q}, T_{\mathcal{K}}^{j-p}(\mathcal{F})\right) .
$$

By Lemma 4.3, and the continuity of $c_{j-p}(f, \mathcal{F})$, each term in the sum is a continuous function, and hence so is $h^{j}(f, \mathcal{F})$.

\section{Two special examples}

This section is largely independent of the full proof of Proposition 6.4. We establish Theorem 2.3 in the special but fundamental case of the Fourier transform, and in a related case which arose during the discussions related to the Polymath8 project [20].

The complications which account for the length of the proof Proposition 6.4, compared with the case of the Fourier transform, are that the

cohomology of the specializations $\mathcal{L}_{\psi(f(x, Y))}$ are not as simple as that of $\mathcal{L}_{\psi(x Y)}$ (for instance, it is not the case in general that $\operatorname{pct}\left(\mathcal{L}_{\psi(f(x, Y))}\right)=0$, as happens in the case of the Fourier transform, see below).

Remark 14.1. - We will not strictly keep track of the fact that the conductor bounds for the Fourier transform are of polynomial size in terms of $\mathbf{c}(\mathcal{F})$, but this is easily checked to follow from the argument.

\subsection{The Fourier transform}

We consider the case

$$
f(X, Y)=X Y \in \mathbf{F}_{q}[X, Y],
$$

and we write $\mathrm{FT}_{\psi}(\mathcal{F})$ for the corresponding transform

$$
\mathrm{FT}_{\psi}(\mathcal{F})=R^{1} p_{1, !}\left(p_{2}^{*} \mathcal{F} \otimes \mathcal{L}_{\psi(X Y)}\right)(1 / 2)
$$

which is up to the Tate twist the "naive" Fourier transform of [9, Chap. 8]. Note that $\mathbf{c}(f)=2$, independently of $q$. We will not need, however to restrict to primes $p>2$.

Let $\mathcal{F}$ be a middle-extension sheaf and

$$
\begin{gathered}
\mathcal{G}=\mathrm{FT}_{\psi}(\mathcal{F}) . \\
-249-
\end{gathered}
$$


By Lemma 4.11 it suffices to show that $\operatorname{rank}(\mathcal{G}), \operatorname{pct}(\mathcal{G})$ and $h_{1}(\mathcal{G})$ are bounded in terms of $\mathbf{c}(\mathcal{F})$.

We start with the rank: by the proper base change theorem, the fiber of $\mathcal{G}$ at $x \in \mathbf{A}^{1}\left(\overline{\mathbf{F}}_{q}\right)$ is

$$
H_{c}^{1}\left(\mathbf{A}^{1} \times \overline{\mathbf{F}}_{q}, \mathcal{F} \otimes \mathcal{L}_{\psi(x Y)}\right) .
$$

From Lemmas 4.4 and 4.3, we already see that the maximum over $x$ of the dimension of these spaces, hence also $\operatorname{rank}(\mathcal{G})$, is bounded in terms of $\mathbf{c}(\mathcal{F})$.

We next claim that $\operatorname{pct}(\mathcal{G})=0$. For this, we use the first spectral sequence of Lemma 7.3 (taking $U=\mathbf{A}^{1}$ there) and apply Proposition 7.2(3) to deduce

$$
\operatorname{pct}(\mathcal{G})=\operatorname{dim} H_{c}^{0}\left(\mathbf{A}^{1} \times \overline{\mathbf{F}}_{q}, \mathcal{G}\right)=\operatorname{dim} H_{c}^{1}\left(\mathbf{A}^{2} \times \overline{\mathbf{F}}_{q}, p_{2}^{*} \mathcal{F} \otimes \mathcal{L}_{\psi(X Y)}\right) .
$$

Let $S \subset \mathbf{A}^{1}$ be the finite set of singularities of $\mathcal{F}$ in $\mathbf{A}^{1}$ and $T=\mathbf{A}^{1} \times S \subset$ $\mathbf{A}^{2}$. The sheaf $\mathcal{M}=p_{2}^{*} \mathcal{F} \otimes \mathcal{L}_{\psi(X Y)}$ is lisse on the dense open set $W=\mathbf{A}^{2}-T$. Applying excision (4.1), we get an exact sequence

$$
\cdots \longrightarrow H_{c}^{1}(\bar{W}, \mathcal{M}) \longrightarrow H_{c}^{1}\left(\mathbf{A}^{2} \times \overline{\mathbf{F}}_{q}, \mathcal{M}\right) \longrightarrow H_{c}^{1}(\bar{T}, \mathcal{M}) \longrightarrow \cdots .
$$

We have

$$
H_{c}^{1}(\bar{W}, \mathcal{M})=0
$$

by (4.4), because $W$ is an affine surface and $\mathcal{M}$ is lisse on $W$. Also, since $T$ is a disjoint union of "horizontal" lines, we have

$$
H_{c}^{1}(\bar{T}, \mathcal{M})=\bigoplus_{y \in S} H_{c}^{1}\left(\mathbf{A}^{1} \times \overline{\mathbf{F}}_{q}, \mathcal{L}_{\psi(y X)}\right) \otimes \mathcal{F}_{y}=0
$$

because $H_{c}^{1}\left(\mathbf{A}^{1} \times \overline{\mathbf{F}}_{q}, \mathcal{L}_{\psi(y X)}\right)=0$ for all $y \in S$ (including $\left.y=0\right)$. The excision exact sequence then gives $H_{c}^{1}\left(\mathbf{A}^{2} \times \overline{\mathbf{F}}_{q}, \mathcal{M}\right)=0$, as claimed.

By Lemma 4.11, we deduce that the conductor of $\mathcal{G}$ is bounded in terms of the conductor of $\mathcal{F}$, and of the invariant

$$
h_{1}(\mathcal{G})=\operatorname{dim} H_{c}^{1}\left(\mathbf{A}^{1} \times \overline{\mathbf{F}}_{q}, \mathcal{G}\right) .
$$

By the first spectral sequence of Lemma 7.3 and Proposition 7.2(1), we get

$$
\operatorname{dim} H_{c}^{1}\left(\mathbf{A}^{1} \times \overline{\mathbf{F}}_{q}, \mathcal{G}\right) \leqslant \operatorname{dim} H_{c}^{2}\left(\mathbf{A}^{2} \times \overline{\mathbf{F}}_{q}, \mathcal{M}\right) .
$$

To compute this last group, we first use the second spectral sequence, which shows that

$$
H_{c}^{2}\left(\mathbf{A}^{2} \times \overline{\mathbf{F}}_{q}, \mathcal{M}\right) \simeq E_{3}^{0,2} \oplus E_{3}^{1,1} \oplus E_{3}^{2,0} \simeq E_{3}^{0,2} \oplus E_{3}^{1,1} .
$$


We have

$$
\begin{aligned}
E_{2}^{1,1} & =H_{c}^{1}\left(\mathbf{A}^{1} \times \overline{\mathbf{F}}_{q}, R^{1} p_{2, !}\left(p_{2}^{*} \mathcal{F} \otimes \mathcal{L}_{\psi(X Y)}\right)\right) \\
& =H_{c}^{1}\left(\mathbf{A}^{1} \times \overline{\mathbf{F}}_{q}, \mathcal{F} \otimes R^{1} p_{2, !} \mathcal{L}_{\psi(X Y)}\right)
\end{aligned}
$$

by the projection formula. But the sheaf $R^{1} p_{2, !} \mathcal{L}_{\psi(X Y)}$ is zero, since the fiber at any $y \in \mathbf{A}^{1}\left(\overline{\mathbf{F}}_{q}\right)$ is

$$
H_{c}^{1}\left(\mathbf{A}^{1} \times \overline{\mathbf{F}}_{q}, \mathcal{L}_{\psi(y X)}\right)=0 .
$$

As for $E_{3}^{0,2}$, it is a subspace of $E_{2}^{0,2}=H_{c}^{0}\left(\mathbf{A}^{1} \times \overline{\mathbf{F}}_{q}, R^{2} p_{2, !}\left(p_{2}^{*} \mathcal{F} \otimes \mathcal{L}_{\psi(X Y)}\right)\right)=H_{c}^{0}\left(\mathbf{A}^{1} \times \overline{\mathbf{F}}_{q}, \mathcal{F} \otimes R^{2} p_{2, !} \mathcal{L}_{\psi(X Y)}\right)$.

The stalk of $R^{2} p_{2, !} \mathcal{L}_{\psi(X Y)}$ at $y \in \overline{\mathbf{F}}_{q}$ is

$$
H_{c}^{2}\left(\mathbf{A}^{1} \times \overline{\mathbf{F}}_{q}, \mathcal{L}_{\psi(y X)}\right)= \begin{cases}\overline{\mathbf{Q}}_{\ell} & \text { if } y=0 \\ 0 & \text { otherwise }\end{cases}
$$

so the sheaf $R^{2} p_{2, !} \mathcal{L}_{\psi(X Y)}$ is punctual and supported at 0 with stalk $\mathcal{F}_{0}$. Hence the dimension of $E_{2}^{0,2}$ is at most the $\operatorname{rank} \operatorname{rank}(\mathcal{F}) \leqslant \mathbf{c}(\mathcal{F})$. Thus we obtain

$$
\operatorname{dim} H_{c}^{2}\left(\mathbf{A}^{2} \times \overline{\mathbf{F}}_{q}, \mathcal{M}\right) \leqslant \mathbf{c}(\mathcal{F}) .
$$

This concludes the proof of Theorem 2.3 for the Fourier transform. We state it formally for convenience:

Corollary 14.2. - Let $\mathbf{F}_{q}$ be a finite field of characteristic $p, \ell \neq$ $p$ a prime number. Let $\psi$ be a non-trivial additive $\ell$-adic character of $\mathbf{F}_{q}$. There exists a function $n \mapsto C(n)$ with positive integral values such that, for any middle-extension sheaf $\mathcal{F}$ on $\mathbf{A}_{\mathbf{F}_{q}}^{1}$, the naive Fourier transform $\mathrm{FT}_{\psi}(\mathcal{F})$ satisfies $\operatorname{pct}\left(\mathrm{FT}_{\psi}(\mathcal{F})\right)=0$ and we have

$$
\mathbf{c}\left(\mathrm{FT}_{\psi}(\mathcal{F})\right) \leqslant C(\mathbf{c}(\mathcal{F})) .
$$

As we already mentioned in the introduction, we obtain in [5, Prop. 8.2] the estimate

$$
\mathbf{c}\left(\mathrm{FT}_{\psi}(\mathcal{F})\right) \leqslant 10 \mathbf{c}(\mathcal{F})^{2}
$$

for $\mathcal{F}$ a Fourier sheaf on $\mathbf{A}_{\mathbf{F}_{p}}^{1}$, using the local study of the Fourier transform, due to Laumon [17]. It is clear that the arguments above can also be used to give a completely effective upper bound.

\section{Remark 14.3.}

(1) A Fourier sheaf is defined to be a middle-extension sheaf which has no subsheaf or quotient sheaf geometrically isomorphic to an ArtinSchreier sheaf $\mathcal{L}_{\psi(a X)}$. For a sheaf which is not of this type, the 
naive Fourier transform is not the right object to consider, but this is of course not due to a failure of continuity.

For instance, if $\mathcal{F}=\mathcal{L}_{\psi(Y)}$ (a typical non-Fourier sheaf!) we have

$$
R^{1} p_{1, !}\left(p_{2}^{*} \mathcal{F} \otimes \mathcal{L}_{\psi(X Y)}\right)=0
$$

since the stalk of this sheaf at $x \in \mathbf{A}^{1}\left(\overline{\mathbf{F}}_{q}\right)$ is

$$
H_{c}^{1}\left(\mathbf{A}^{1} \otimes \overline{\mathbf{F}}_{q}, \mathcal{L}_{\psi((1+x) Y)}\right)=0
$$

for all values of $x$. This certainly has bounded conductor!

(2) For Fourier sheaves, other properties of the Fourier transform are established, relatively elementarily, in [9, 8.2.5, 8.4.1]: the Fourier transform is again a Fourier sheaf, and the Fourier transform of a geometrically irreducible Fourier sheaf is again geometrically irreducible.

We can deduce a version of the irreducibility property (which suffices in many applications) from the diophantine irreducibility criterion of Lemma 5.13. Indeed, if $\mathcal{F}$ is a middle-extension Fourier sheaves which is pointwise pure of weight 0 , we have the discrete Plancherel formula

$$
\frac{1}{q^{\nu}} \sum_{x \in \mathbf{F}_{q^{\nu}}}\left|t_{\mathcal{F}}\left(x, q^{\nu}\right)\right|^{2}=\frac{1}{q^{\nu}} \sum_{t \in \mathbf{F}_{q^{\nu}}}\left|t_{\mathrm{FT}_{\psi}(\mathcal{F})}\left(t, q^{\nu}\right)\right|^{2}
$$

for $\nu \geqslant 1$. The Fourier transform $\mathrm{FT}_{\psi}(\mathcal{F})$ is mixed of weight $\leqslant 0$ by the Riemann Hypothesis (in fact, it is known to be pure of weight 0 , but this is again a deeper fact), hence Lemma 5.13 implies that $\mathcal{F}$ is geometrically irreducible if and only if the weight 0 part of $\mathrm{FT}_{\psi}(\mathcal{F})$ is geometrically irreducible.

\subsection{The Polymath8 kernel}

We next consider another example discussed in the blog of the PoLYMATH8 project, which we will reduce to a Fourier transform. We let

$$
f=\frac{1}{X(X+Y)}+h Y
$$

where $h \in \mathbf{F}_{q}^{\times}$is a parameter, and we wish to bound the conductor of

$$
R^{1} p_{1, !} \mathcal{L}_{\psi(f)}(1 / 2),
$$

i.e., the corresponding transform of the trivial sheaf, by a constant (independent of $q$ ).

We outline the steps that prove such a bound, leaving some details to the reader. 
- It is equivalent to bound the conductor of

$$
R^{1} p_{1, !} \mathcal{L}_{\psi(g)}(1 / 2)
$$

where $g=(X Y)^{-1}+h Y-h X$ (applying the automorphism $(X, Y) \mapsto$ $(X, X+Y))$. By the projection formula, we have

$$
R^{1} p_{1, !} \mathcal{L}_{\psi(g)}(1 / 2)=\mathcal{L}_{\psi(-h X)} \otimes \mathcal{G},
$$

where

$$
\mathcal{G}=R^{1} p_{1, !} \mathcal{L}_{\psi(h)}(1 / 2), \quad h=\frac{1}{X Y}+h Y .
$$

By Lemma 4.4, it is enough to estimate the conductor of $\mathcal{G}$.

- Note that the trace function of $\mathcal{G}$ is

$$
\frac{1}{q^{1 / 2}} \sum_{y \neq 0} \psi\left(\frac{1}{x y}+h y\right)=\frac{1}{q^{1 / 2}} \sum_{v \neq 0} \psi\left(\frac{1}{v}+\frac{h}{x} v\right)
$$

for $x \neq 0$, which is visibly a normalized Kloosterman sum with parameter $h / x$. Let

$$
\pi:\left\{\begin{array}{l}
\mathbf{G}_{m} \times \mathbf{G}_{m} \rightarrow \mathbf{G}_{m} \times \mathbf{G}_{m} \\
(x, y) \mapsto\left(h x^{-1}, x y\right)
\end{array}\right.
$$

and

$$
\nu:\left\{\begin{array}{l}
\mathbf{G}_{m} \rightarrow \mathbf{G}_{m} \\
x \mapsto h x^{-1}
\end{array}\right.
$$

Then the analogue of the change of variable $(u, v)=\pi(x, y)=$ $(h / x, x y)$ that establishes this identity is the isomorphism

$$
\nu^{*} R^{1} p_{1, !} \mathcal{L}_{\psi\left(V^{-1}+U V\right)} \simeq R^{1} p_{1, !} \mathcal{L}_{\psi\left((X Y)^{-1}+h Y\right)}
$$

of sheaves over the multiplicative group $\mathbf{G}_{m}=\mathbf{A}^{1}-\{0\}$ over $\mathbf{F}_{q}$, which is a consequence of the isomorphism $\pi^{*} \mathcal{L}_{\psi\left(V^{-1}+U V\right)} \simeq \mathcal{L}_{\psi\left((X Y)^{-1}+Y\right)}$.

Note that

$$
R^{1} p_{1, !} \mathcal{L}_{\psi\left(V^{-1}+U V\right)}(1 / 2)=\mathrm{FT}_{\psi}\left(\mathcal{L}_{\psi\left(V^{-1}\right)}\right),
$$

which has bounded conductor independently of $q$. Since $\nu$ is an automorphism, and since the dimensions of the stalk of $\mathcal{G}$ at 0 is bounded, it follows from the fact that $\mathcal{G}$ coincides with $\left(\nu^{-1}\right)^{*} \mathrm{FT}_{\psi}\left(\mathcal{L}_{\psi\left(V^{-1}\right)}\right)$ on $\mathbf{G}_{m}$ that the conductor of $\mathcal{G}$ is bounded for all $q$, as desired.

Remark 14.4. - The Fourier transform of $\mathcal{L}_{\psi\left(X^{-1}\right)}$ is the Kloosterman sheaf (in one variable), that was originally defined by Deligne. See [9] for its properties, and generalizations to more than one variable. 


\section{Bibliography}

[1] J. B. Conrey \& H. IwAniec, "The cubic moment of central values of automorphic L-functions", Ann. Math. 151 (2000), no. 3, p. 1175-1216.

[2] P. Deligne, Cohomologie étale, Lecture Notes in Mathematics, vol. 569, Springer, 1977, S.G.A, $4 \frac{1}{2}$.

[3] - "La conjecture de Weil. II", Publ. Math., Inst. Hautes Étud. Sci. 52 (1980), p. 137-252.

[4] É. Fouvry, E. Kowalski \& P. Michel, "Algebraic trace weights over the primes", Duke Math. J. 163 (2014), no. 9, p. 1683-1736.

[5] - "Algebraic twists of modular forms and Hecke orbits", Geom. Funct. Anal. 25 (2015), no. 2, p. 580-657.

[6] - "On the exponent of distribution of the ternary divisor function", Mathematika 61 (2015), no. 1, p. 121-144.

[7] L. Fu, Etale cohomology theory, Nankai Tracts in Mathematics, vol. 13, World Scientific, 2011.

[8] N. M. Katz, Sommes exponentielles, Astérisque, vol. 79, Société Mathématique de France, 1980.

[9] - Gauss sums, Kloosterman sums, and monodromy groups, Annals of Mathematics Studies, no. 116, Princeton University Press, 1988.

[10] - Exponential Sums and Differential Equations, Annals of Mathematics Studies, vol. 124, Princeton University Press, 1990.

[11] —, "Affine cohomological transforms, perversity, and monodromy", J. Am. Math. Soc. 6 (1993), no. 1, p. 149-222.

[12] — Rigid local systems, Annals of Mathematics Studies, vol. 139, Princeton University Press, 1996.

[13] - "L-functions and monodromy: four lectures on Weil II", Adv. Math. 160 (2001), no. 1, p. 81-132.

[14] - "Sums of Betti numbers in arbitrary characteristic", Finite Fields Appl. 7 (2001), no. 1, p. 29-44.

[15] E. Kowalski, P. Michel \& W. Sawin, "Bilinear forms with Kloosterman sums and applications", Ann. Math.(2) 186 (2017), no. 2, p. 413-500.

[16] G. Laumon, "Semi-continuité du conducteur de Swan (d'après P. Deligne)", in Caractéristique d'Euler-Poincaré, Astérisque, vol. 83, Société Mathématique de France, 1981, p. 173-219.

[17] — , "Transformation de Fourier, constantes d'équations fonctionnelles et conjecture de Weil", Publ. Math., Inst. Hautes Étud. Sci. 65 (1987), p. 131-210.

[18] J. S. Milne, Étale cohomology, Princeton Mathematical Series, vol. 33, Princeton University Press, 1980.

[19] I. Petrow \& M. P. Young, "The Weyl bound for Dirichlet $L$-functions of cube-free conductor", Ann. Math. 192 (2020), no. 2, p. 437-486.

[20] G. H. J. Polymath, "New equidistribution estimates of Zhang type", Algebra Number Theory 8 (2014), no. 9, p. 2067-2199.

[21] J. J. Rotman, An introduction to homological algebra, 2nd ed., Springer Universitext, 2009.

[22] W. SAwin, "Quantitative sheaf theory", drafted by A. Forey, J. Fresán and E. Kowalski, https://arxiv.org/abs/2101.00635. 